\title{
Uranus in Northern Midspring: Persistent Atmospheric Temperatures and Circulations Inferred from Thermal Imaging
}

\author{
Michael T. Roman $^{1}$ (D), Leigh N. Fletcher ${ }^{1}$ (D), Glenn S. Orton ${ }^{2}$ (D), Naomi Rowe-Gurney ${ }^{1}$ (D), and Patrick G. J. Irwin ${ }^{3}$ (D) \\ ${ }^{1}$ University of Leicester, School of Physics and Astronomy, University Road, Leicester, LE1 7RH, UK; mr359@le.ac.uk \\ 2 Jet Propulsion Laboratory, MS 183-501, Pasadena, CA 91109, USA \\ ${ }^{3}$ University of Oxford, Atmospheric, Oceanic, and Planetary Physics, Department of Physics, Clarendon Laboratory, Parks Road, Oxford OX1 3PU, UK \\ Received 2019 October 21; revised 2019 November 27; accepted 2019 November 27; published 2020 January 10
}

\begin{abstract}
We present results from mid-infrared imaging of Uranus at wavelengths of 13.0 and $18.7 \mu \mathrm{m}$, sensing emission from the stratosphere and upper troposphere, acquired using the VISIR instrument at the Very Large Telescope, 2018 September 4-October 20. Using a combination of inverse and forward modeling, we analyze these northern midspring $\left(L_{s} \sim 46^{\circ}\right)$ images and compare them to archival data to assess seasonal changes since the 1986 southern solstice and subsequent equinox. We find the data are consistent with little change $(<0.3 \mathrm{~K})$ in the upper tropospheric temperature structure, extending the previous conclusions of Orton et al. well past equinox, with only a subtle increase in temperature at the emerging north pole. Additionally, spatial-temporal variations in $13 \mu \mathrm{m}$ stratospheric emission are investigated for the first time, revealing meridional variation and a hemispheric asymmetry not predicted by models. Finally, we investigate the nature of the stratospheric emission and demonstrate that the observed distribution appears related and potentially coupled to the underlying tropospheric emission six scale heights below. The observations are consistent with either midlatitude heating or an enhanced abundance of acetylene. Considering potential mechanisms and additional observations, we favor a model of acetylene enrichment at midlatitudes resulting from an extension of the upper tropospheric circulation, which appears capable of transporting methane from the troposphere, through the cold trap, and into the stratosphere for subsequent photolysis to acetylene.
\end{abstract}

Unified Astronomy Thesaurus concepts: Atmospheric circulation (112); Uranus (1751); Atmospheric science (116); Planetary atmospheres (1244); Planetary science (1255); Outer planets (1191)

\section{Introduction}

The atmosphere of Uranus is subject to a unique pattern of seasonal forcing. Due to the planet's $98^{\circ}$ obliquity, nearly all latitudes on Uranus experience seasonally extended periods of total daylight and darkness. Averaged over a full orbital period, this seasonal cycle results in more solar energy being deposited annually at the poles than at the equator, contrary to the other solar system planets (Friedson \& Ingersoll 1987; Conrath et al. 1990; Moses et al. 2018). While observations of reflected light clearly show a seasonal cycle in the tropospheric hazes at polar latitudes (e.g., Karkoschka 2001; Rages et al. 2004; Irwin et al. 2010; Roman et al. 2018; Toledo et al. 2018; Lockwood 2019; Sromovsky et al. 2019), the effects of this peculiar seasonal forcing on Uranus' temperature field, atmospheric chemistry, and circulations have yet to be fully observed or modeled.

Given the pattern of solar forcing, early radiative-convective modeling suggested the summer pole of Uranus should be 1.5-6 K warmer than the equator and winter pole (Wallace 1983; Friedson \& Ingersoll 1987). The first and most complete measurements of the pole-to-pole variation in thermal emission from Uranus came in 1986 with the Infrared Interferometer Spectrometer and Radiometer (IRIS; Hanel et al. 1986) aboard Voyager 2. Spectral data from the flyby were inverted to yield upper tropospheric (70-400 mbar) temperatures near the time of the southern summer solstice. Contrary to expectations, these data showed the warmest temperatures at the equator and poles, with colder temperatures at midlatitudes (Flasar et al. 1987; Conrath et al. 1990; Orton et al. 2015), interpreted by Flasar et al. (1987) as an indication of an organized atmospheric circulation, with upwelling at midlatitudes and downwelling at the equator and high latitudes producing adiabatic cooling and heating, respectively. Although the coldest portions of the northern winter hemisphere were slightly colder $(\sim 1 \mathrm{~K})$ than the equivalent latitudes in the summer hemisphere, the two poles were roughly the same temperature at the tropopause, and the summer pole was inferred to be only marginally warmer in the lower stratosphere (Orton et al. 2015).

Flasar et al. (1987) and Conrath et al. (1990) proposed that the inferred lack of seasonal response could be largely explained by atmospheric radiative time constants $\left(t_{r}\right)$ that were relative to Uranus' orbital period $\left(t_{o}\right)$, specifically such that $2 \pi t_{r} / t_{o} \gg 1$. Using a radiative-convective-dynamical model, Conrath et al. (1990) suggested that a radiative time constant of $130 \mathrm{yr}$ would appropriately dampen the atmospheric temperature response by a factor of 10 , resulting in seasonal variation of only $\sim 1 \mathrm{~K}$, lagging roughly a full season behind the instantaneous solar forcing. They noted, however, that this would not explain the hemispheric asymmetries, inconsistent with symmetric equinoctial forcing, present in the seasonally lagged solstitial data.

One season later, around the time of the 2007 southern autumnal equinox $\left(L_{s} \sim 0^{\circ}\right)$, Uranus' spatially resolved radiance was evaluated by Orton et al. (2015) sing thermal ground-based imaging and compared to the earlier Voyager measurements. The study found very little if any change $(<0.4 \mathrm{~K})$ in the implied thermal structure from the southern summer solstice to the southern autumnal equinox, including the noted asymmetry at midlatitudes, from which it was concluded that upper tropospheric radiative time constants must be no less than $\sim 330 \mathrm{yr}$. At that value, the phase lag would have essentially reached a maximum of roughly a season as the amplitude of the seasonal temperature variations diminishes (Conrath et al. 1990). 
The long radiative time constants suggested by the data are apparently in conflict with values calculated using radiativetransfer modeling. Theoretical radiative time constants as a function of height for the outer planet atmospheres have been reevaluated recently by $\mathrm{Li}$ et al. (2018) using a radiativetransfer model with improved correlated-k gas opacities and state-of-the-art photochemical models. For Uranus, Li et al. (2018) calculated radiative time constants with values less than those of Conrath et al. (1990), ranging from roughly 15 to $70 \mathrm{yr}$ at 400 to 70 mbar pressures. The reason for this apparent discrepancy between theoretically predicted values and those inferred from observations are unknown, but it may possibly indicate errors in the assumed abundances, distributions, and/ or opacities of radiatively active hydrocarbons.

The distribution of hydrocarbons produced photochemically from the seasonally varying solar flux on Uranus has been recently modeled by Moses et al. (2018). Though chemical abundances were constrained by disk-averaged spectra of Uranus from Spitzer at northern vernal equinox (Orton et al. 2014b), the theoretically predicted seasonal variations remain unverified by data. Likewise, the spatial distributions of hydrocarbon species remain largely unknown given the nearly complete absence of spatially resolved images and spectra in the literature. As Moses et al. (2018) note, their model does not include the potentially significant role of general circulation, which could potentially produce gradients in the methane abundance and consequent photolytic products. As we will show, meridional variations in hydrocarbons or temperature are indeed required for modeling the observed disk-resolved emission associated with acetylene, suggesting a significant dynamical link between the troposphere and stratosphere extending over several pressure scale heights.

To this end, we present and analyze newly acquired midinfrared images of Uranus-halfway through Uranus' northern spring with the north pole now fully in view for the first time in the era of thermal imaging - to investigate temporal changes in atmospheric temperatures and circulation. New and archival data are introduced in Section 2, followed by a description of our methods for analysis in Section 3 and results in Section 4. We conclude with a discussion of the implications of our results in Section 5 and a brief summary of the conclusions in Section 6.

\section{Data}

\subsection{New Observations: 2018 Images}

Images of Uranus were acquired in 2018 September and October using the mid-infrared VISIR instrument (Lagage et al. 2004; Kerber et al. 2014) at the European Southern Observatory's (ESO) Very Large Telescope (VLT, UT3). Two separate filters were used: Q2, with a central wavelength of $18.7 \mu \mathrm{m}\left(534.2 \mathrm{~cm}^{-1}\right)$ and a full-width at half maximum of $0.88 \mu \mathrm{m}$; and NeII_2, centered at $13.0 \mu \mathrm{m}\left(769.2 \mathrm{~cm}^{-1}\right)$ with a FWHM of $0.22 \mu \mathrm{m}$.

The $18.7 \mu \mathrm{m}$ filter (Q2) is sensitive to the continuum emission from atmospheric hydrogen and thus serves as a probe of upper tropospheric temperatures with a peak contribution from 200 mbar at nadir geometry (contribution functions are provided in Appendix A, Figure 14). Images show enhanced emission at the equator and pole relative to midlatitudes, qualitatively consistent with inferences of midlatitudinal cooling in the upper troposphere dating back to the Voyager era (Flasar et al. 1987; Conrath et al. 1990; Orton et al. 2015).
In contrast, the $13.0 \mu \mathrm{m}$ filter (NeII_2) is dominated by thermal emission associated with stratospheric acetylene with maximum contributions from pressures near 0.2 mbar. The $13.0 \mu \mathrm{m}$ images show brighter emission at middle and high latitudes compared to the equator. Because $\mathrm{C}_{2} \mathrm{H}_{2}$ is a minor and likely variable species (Moses et al. 2018), variation in the $13.0 \mu \mathrm{m}$ emission can be attributed to variation in either the $\mathrm{C}_{2} \mathrm{H}_{2}$ abundance, the stratospheric temperatures, or a combination of the two quantities.

Jointly, these images reveal tropospheric temperatures and stratospheric emission from Uranus' northern hemisphere in midspring (subsolar latitudes of $44^{\circ}-45^{\circ}$ ), including unprecedented views of Uranus' north pole and the first-ever images of Uranus' rings in the mid-infrared. Analysis of the rings based on these images is presented in Molter et al. (2019). Data were collected in $\sim 45$ minute blocks on six different nights (three nights for each filter) and reduced using ESO's pipeline of standard infrared chopping and nodding techniques. The resulting images (see Figure 1) were flux calibrated by comparison to observed standard stars. We assumed a systematic error of up to $30 \%$ in radiance due to fluctuations in sky brightness and up to $20 \%$ due to uncertainties in the stellar fluxes (Dobrzycka \& Vanzi 2008). Random errors were estimated from the standard deviation of the background sky. To improve the signal-to-noise ratio $(\mathrm{S} / \mathrm{N})$ in each filter, the calibrated images from each night were weighted by the inverse of their squared random errors and combined to yield the final, absolutely calibrated mean image for each filter. A summary of our 2018 observations is provided in Table 1.

The random noise was significant in both images given the weak signal from Uranus' cold atmosphere. For individual images, we estimate an average $\mathrm{S} / \mathrm{N}$ for individual pixels upon the disk to be as low as $\sim 1.2$ at $18.7 \mu \mathrm{m}$ and $\sim 0.9$ at $13.0 \mu \mathrm{m}$. Combining the nights increased these $\mathrm{S} / \mathrm{Ns}$ to $\sim 2.8$ and $\sim 1.6$, respectively, varying with signal across the disk. Zonal averaging and meridional binning were employed to significantly enhance the $\mathrm{S} / \mathrm{N}$ in computed latitudinal profiles.

\subsection{Archival Data}

To evaluate temporal changes in the $18.7 \mu \mathrm{m}$ images, we compared our new data to Voyager data (the oldest spatially resolved thermal data of Uranus available) following the techniques of Orton et al. (2015), as described in Section 3. In the case of the $13.0 \mu \mathrm{m}$ stratospheric emission, the Voyager IRIS instrument was unfortunately not sensitive enough to provide measurements, and so changes in the NeII_2-band images were evaluated using VLT-VISIR images dating from near equinox (Orton et al. 2018; see Figure 2).

The near-equinoctial data included two consecutive nights of $13 \mu \mathrm{m}$ imaging from 2009, but no contemporaneous $18.7 \mu \mathrm{m}$ images were available. We instead substituted a similarly sensitive $19.5 \mu \mathrm{m}$ image (Q3 filter) to define the tropospheric emission when performing the stratospheric retrievals discussed in Section 3. Due to a previously greater plate scale on VISIR (0.075 versus $0.0453 \mathrm{arcsec} /$ pixel), 2009 images were acquired at a coarser spatial resolution than the 2018 images, but they otherwise present equivalent measurements at a different time and viewing geometry. The latitudinal coverage and relative plate scale for both the 2009 and 2018 observations can be seen in Figure 3. 


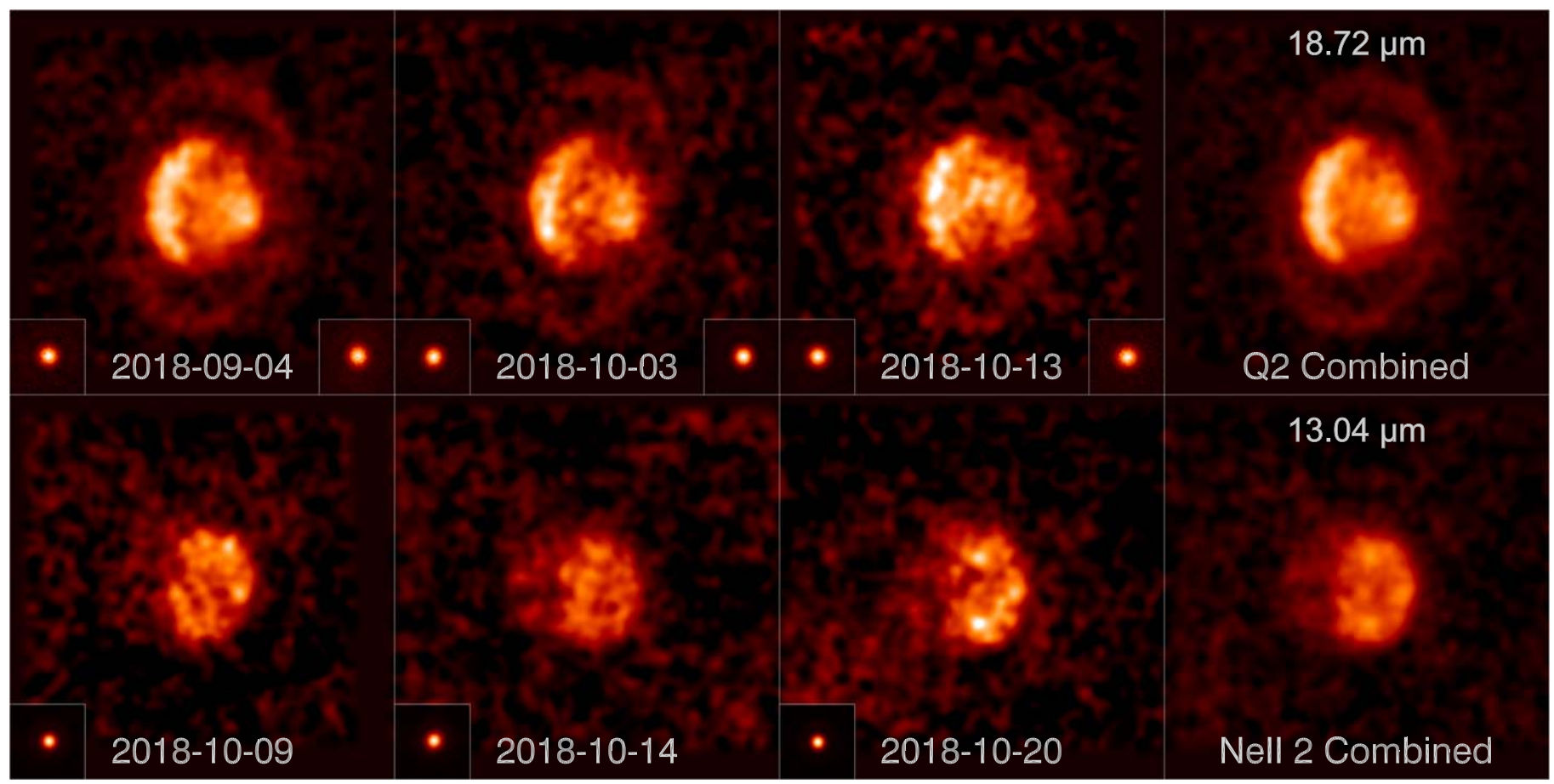

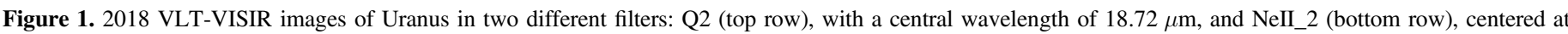

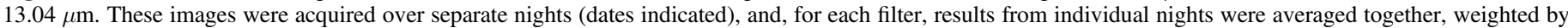

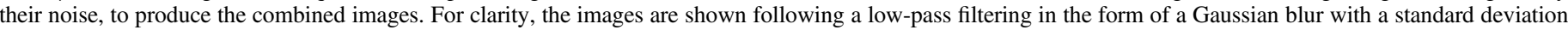

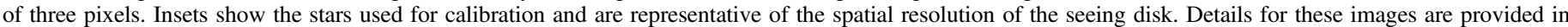

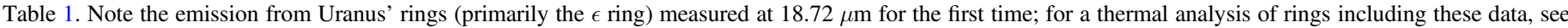
Molter et al. (2019)

Table 1

Summary of 2018 Observations

\begin{tabular}{|c|c|c|c|c|c|}
\hline Filter (Wavelength) & Date (UT) & Time (UTC) & Airmass & Calibration Star & Sub-observer (Solar) Latitude \\
\hline \multirow[t]{3}{*}{ Q2 [18.72 $\mu \mathrm{m}]$} & 2018 Sep 4 & $8: 14-9: 03$ & $1.249-1.316$ & HD 013596, HD 009692 & $45.3(43.0)$ \\
\hline & 2018 Oct 3 & $5: 15-5: 54$ & $1.236-1.255$ & HD 008498, HD 010380 & $44^{\circ} 4(43.4)$ \\
\hline & 2018 Oct 13 & $5: 32-6: 04$ & $1.240-1.272$ & HD 011353, HD 040808 & $44^{\circ} 0(43.5)$ \\
\hline \multirow[t]{3}{*}{ NeII_2 [13.04 $\mu \mathrm{m}]$} & 2018 Oct 09 & $5: 13-5: 55$ & $1.238-1.245$ & HD 008498 & $44.2(43.4)$ \\
\hline & 2018 Oct 14 & $4: 51-5: 39$ & $1.234-1.237$ & HD 008498 & $44^{\circ} 0(43.5)$ \\
\hline & 2018 Oct 20 & $4: 49-5: 36$ & $1.233-1.271$ & HD 011353 & $43.8(43.5)$ \\
\hline
\end{tabular}

Note. The VISIR instrument in 2018 had a plate scale of $0.0453 \mathrm{arcsec} /$ pixel.

Data from each night were collected in blocks, reduced using the ESO pipeline, and combined to yield nightly averages. We initially attempted to calibrate these images using standard calibration stars, but we found resulting radiances to be $10 \%-40 \%$ less than the disk-integrated values from the more reliable Spitzer data acquired less than two years earlier (Orton et al. 2014a). Ultimately, given the uncertainty in the ground-based calibrations and the relative contemporaneity of the space-based Spitzer observations, we chose to scale the 2009 images such that their disk-integrated radiances equaled the Spitzer values at the equivalent central wavelengths. Details on these observations are found in Table 2 .

The 2009 images reveal tropospheric temperatures and stratospheric emission near equinox $\left(L_{s} \sim 7^{\circ}\right)$, with subsolar latitudes of $\sim 7^{\circ}$. S/Ns were $\sim 6-7$ for the $19.5 \mu \mathrm{m}$ and $\sim 3-4$ for the $13.0 \mu \mathrm{m}$ images. Though the systematic uncertainties for Spitzer are less than $10 \%$, we conservatively estimate systematic errors as high as $20 \%$ to account for changes in viewing geometry and radiance in the intervening $1.5 \mathrm{yr}$.

\section{Methods}

\subsection{Comparing Voyager Tropospheric Temperatures to $Q 2$ Images}

To evaluate temporal changes in the tropospheric emission, we followed the method of Orton et al. (2015), which compared synthetic images, derived from Voyager/IRIS spectra, to a collection of ground-based imaging data. In that work, $Q$-band images (including $18.7 \mu \mathrm{m}$ and $19.5 \mu \mathrm{m}$ VISIR data from 2006 and 2009, respectively) were analyzed to assess changes in the meridional distribution of upper tropospheric temperatures between the 1986 Voyager encounter (roughly coinciding with Uranus' southern summer solstice) and the following equinox (southern autumn) in 2007. No significant changes were detected (Orton et al. 2015). Using a similar approach, we compared our 2018 images to the 1986 Voyager data to evaluate potential changes over a longer interval.

First, we forward-modeled emission from the temperatures inferred from the Voyager measurements to produce a synthetic 


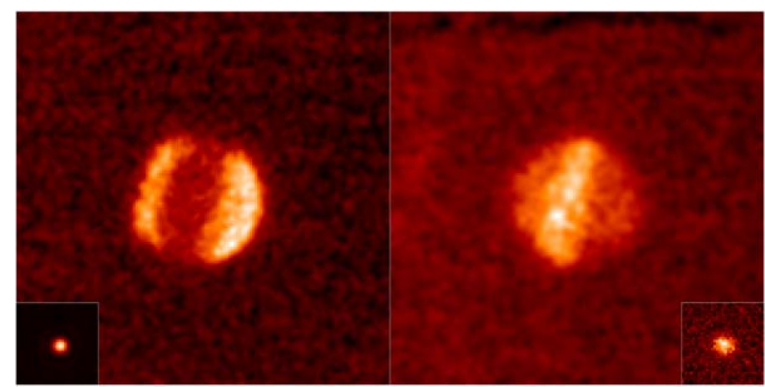

Figure 2. Mean near-equinoctial VLT-VISIR observations of Uranus (Orton et al. 2018) used for our retrievals to evaluate temporal changes in the $13.0 \mu \mathrm{m}$ data. (Left) Mean NeII_2 (13.04 $\mu \mathrm{m}$ ) image from 2009 August 5 and 6. (Right) Mean Q3 $(19.5 \mu \mathrm{m})$ image from 2009 August 3, sensing upper tropospheric depths similar to the Q2 $(18.7 \mu \mathrm{m})$ images.

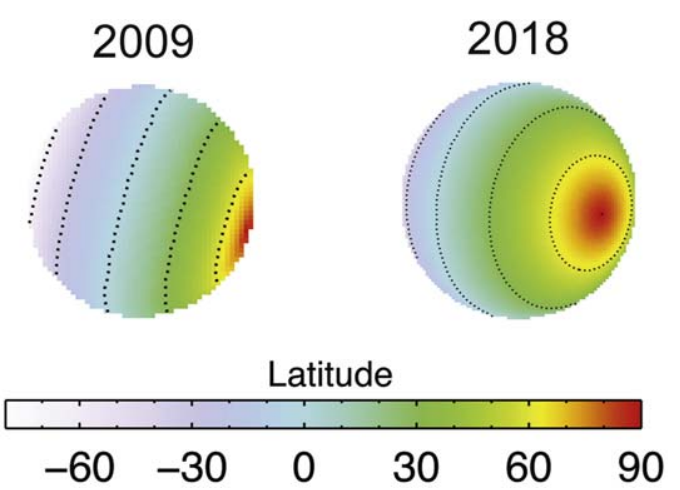

Figure 3. Latitudinal coverage for the 2009 (left) and 2018 (right) observations. Latitudes are indicated by the color bar. Dotted lines additionally mark latitudes of $-60^{\circ},-30^{\circ}, 0^{\circ}, 60^{\circ}$, and $90^{\circ}$. The 2018 map has a finer pixel resolution relative to the 2009 map, due to the improved plate scale.

image of Voyager-era emission with 2018 geometry-effectively what we would have observed at $18.7 \mu \mathrm{m}$ in 2018 if the upper tropospheric temperatures near 200 mbar remained unchanged since 1986. These temperatures were taken directly from Orton et al. (2015), which reanalyzed Voyager 2/IRIS spectra acquired over the four days surrounding the closest approach to Uranus on 1986 January 24. These data covered both the southern and northern hemispheres as the spacecraft passed Uranus at distances ranging from 4.2 to 112 Uranus radii. Spectra were inverted to yield temperatures as a function of latitude and pressure from 70 to 400 mbar and then extended to lower pressures based on Spitzer observations (Orton et al. 2014a). We refer the reader to Orton et al. (2015) for further details on how the Voyager temperatures were retrieved.

We then convolved the forward-modeled images of radiance with point spread functions (PSFs) determined from the corresponding stellar images to model the effects of atmospheric seeing and instrumental diffraction. To account for any errors associated with imperfect navigation and stacking of our three images, we created a synthetic image for each of the three individual observations and combined them in precisely the same way as we had done for the data. Finally, zonal averages (binned $10^{\circ}$ in latitude to improve the $\mathrm{S} / \mathrm{N}$ of the data) were extracted from both the synthetic and real images, converted from units of radiance into brightness temperatures, and compared as a function of latitude (e.g., see Figures 6 and 7).

Because Orton et al. (2015) found no significant changes in the upper tropospheric temperatures between the 1986 solstice and the 2007 equinox (using a wider range of VISIR data and
Table 2

Summary of 2009 Observations

\begin{tabular}{lccc}
\hline \hline Filter [Wavelength] & Date (UT) & Time (UTC) & Airmass \\
\hline Q3 [19.50 $\mu \mathrm{m}]$ & 2009 Aug 3 & $05: 40-06: 12$ & $1.164-1.247$ \\
& $06: 20-06: 53$ & $1.104-1.151$ \\
& $06: 56-07: 19$ & $1.086-1.102$ \\
& $07: 45-08: 10$ & $1.082-1.091$ \\
& $08: 21-08: 44$ & $1.098-1.125$ \\
NeII_2 [13.04 $\mu \mathrm{m}]$ & $08: 46-09: 10$ & $1.167-1.152$ \\
& & $09: 13-09: 35$ & $1.173-1.272$ \\
& & $09: 38-10: 01$ & $1.237-1.229$ \\
& & $06: 56-07: 19$ & $1.083-1.095$ \\
& $07: 32-07: 56$ & $1.081-1.087$ \\
& $08: 10-08: 33$ & $1.097-1.122$ \\
& & $08: 46-09: 10$ & $1.142-1.188$ \\
& & $07: 26-07: 59$ & $1.081-1.091$ \\
& & $08: 02-08: 32$ & $1.092-1.125$ \\
& & $08: 39-09: 13$ & $1.132-1.201$ \\
& & $09: 15-09: 49$ & $1.207-1.323$ \\
\hline
\end{tabular}

Note. The VISIR instrument in 2009 had a plate scale of $0.0750 \mathrm{arcsec} /$ pixel. Standard stars were observed for PSF determination, but photometric calibration was performed by comparison to Spitzer values (Orton et al. 2014a). HD 12524, HD 5112, and HD 216149 were observed in the NEII_2 filter; HD 224630, HD 220954, HD 220009, and HD 198048 were observed in the Q3 filter. For all images, the sub-observer latitude was $\sim 8.8^{\circ}$, and the subsolar latitude was $\sim 6.7^{\circ}$.

the same radiative-transfer code applied in the present study), we chose to only assess changes between the 1986 Voyager data and our $201818.7 \mu \mathrm{m}$ data.

\subsection{Evaluating Stratospheric Changes}

Evaluating temporal changes at the roughly 0.2-mbar heights sensed by the $13.0 \mu \mathrm{m}$ (NeII_2) filter (see contribution functions in Appendix A, Figure 14) required a more complicated approach combining radiative-transfer forward modeling and retrievals. Because the Voyager/IRIS measurements were insensitive to the weak emission at $13 \mu \mathrm{m}$, we drew upon archival $13 \mu \mathrm{m}$ images from 2009 for comparison (see Section 2.2). However, given the nine year difference between the 2018 and 2009 images, a direct, quantitative comparison required accounting for differences in the observing geometry that can affect the observed radiance as a function of emission angle. To account for this, we took the following approach: first, starting with a prior 1D model of Orton et al. (2014a), we retrieved zonally averaged temperature and acetylene profiles from the observations, accounting for the dependence on emission angle, and used these retrieved values to create a model of the atmosphere (i.e., the temperature and acetylene as a function of pressure and latitude). Then, as we had done with the Voyager temperatures, we computed the radiances emitted from this atmospheric model at different desired emission angles, resulting in a synthetic image (i.e., a forward model) of the planetary emission at a different viewing geometry.

In practice, gradients in the observed emission in our data can be equally explained by gradients in the stratospheric temperatures or the acetylene mixing ratios. We performed retrievals in which one of the two parameters (either temperature or acetylene) was held fixed above the 1 mbar height (assuming values of Orton et al. 2015 and Moses et al. 2018) while the other parameter was free to vary. We found $1 \mathrm{mbar}$ to be a reasonable pressure boundary for separating the $13 \mu \mathrm{m}$ filter 


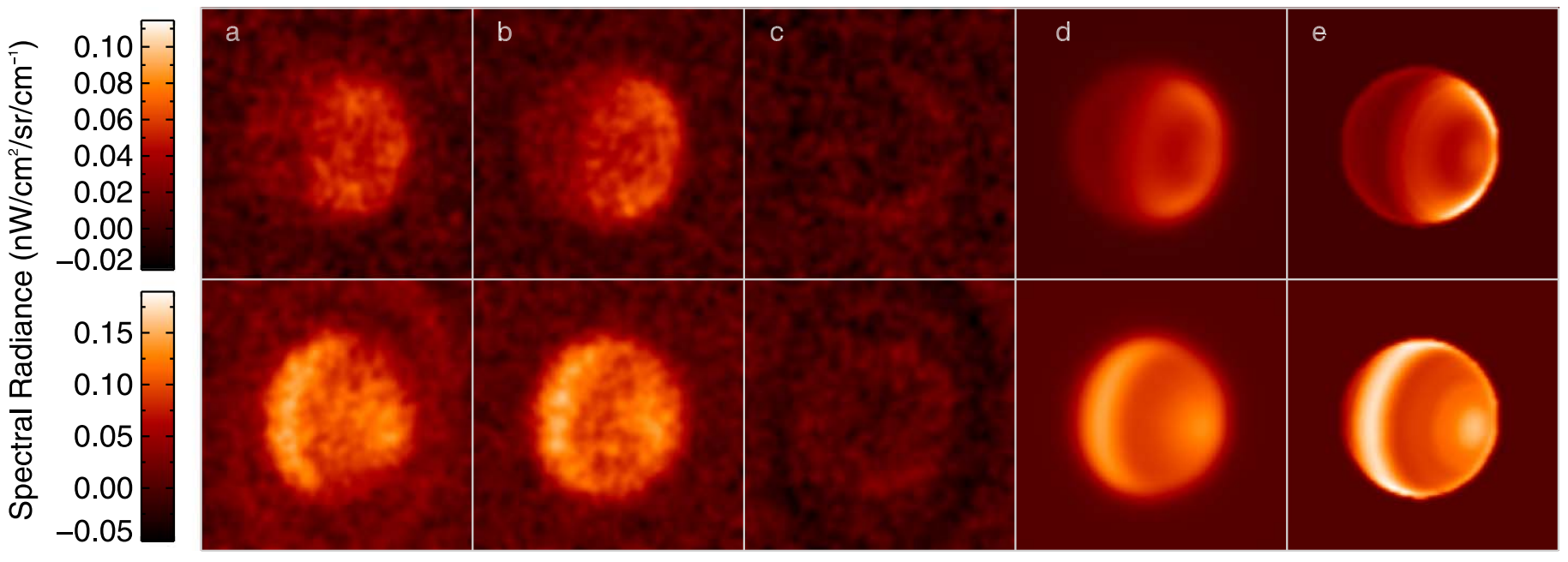

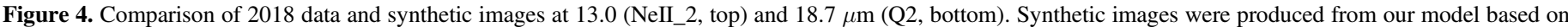

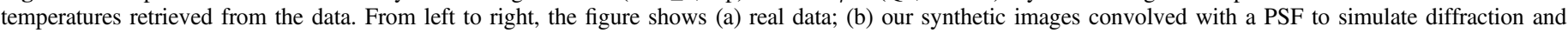

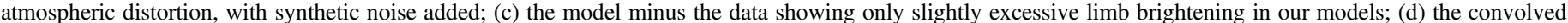

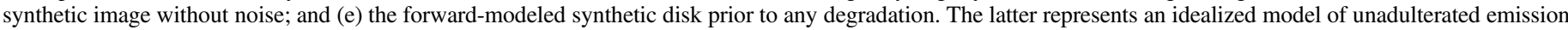

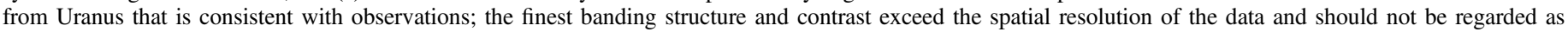
physically significant. Zonal averages extracted from panels (d) and (e) correspond to the solid and dashed lines in Figure 6, respectively.

contribution from the deeper $18.7 \mu \mathrm{m}$ contribution. Although the $13 \mu \mathrm{m}$ contribution function showed some sensitivity to pressures greater than 1-mbar, enhancement in parameters at these deeper levels was found to produce excessive limb darkening, strongly inconsistent with the center-to-limb variation in the observations. Regardless, retrieved models of either stratospheric temperatures or acetylene worked equally well for forward modeling the emission.

All retrievals were performed on the contemporaneous image pairs using an optimal estimation retrieval algorithm, NEMESIS (Irwin et al. 2008). Calculations used collision-induced opacity based on Fletcher et al. (2018), $\mathrm{C}_{2} \mathrm{H}_{2}$ and other hydrocarbon line data from the GEISA-2003 compilation (Jacquinet-Husson et al. 2011), and precomputed k-distributions based on the VISIR filter properties. Additional details of the retrieval and forward modeling process are included in Appendix B.

With the retrieved parameters, we updated the prior $1 \mathrm{D}$ model (as a function of pressure) and created a 2D model (as a function of pressure and latitude). For latitudes beyond the observed domain, values were simply extended from the edge of the domain. The retrieved atmospheric models were forward modeled at different viewing geometries using NEMESIS and the same spectroscopic data as used for the retrievals.

To validate our modeling approach, synthetic images using unchanged viewing geometries were also created; these forwardmodeled emission were convolved with appropriate PSFs, degraded with synthetic noise, and compared to the data. The added synthetic noise was modeled as an array of pseudorandom numbers from a normal (Gaussian) distribution with a standard deviation equal to the standard deviation of the sky in the images. As Figures 4 and 5 show, the synthetic images created from the forward models simulate the data very accurately, providing confidence in our characterization of the radiance, PSF, and random noise. The only significant deviation is near the limb in modeled $13.0 \mu \mathrm{m}$ images, which appears only marginally brighter than the data. This likely suggests that the upper stratospheric temperatures are increasing too rapidly with increasing height in the adopted temperature model (Orton et al. 2014a) at altitudes above 0.1 mbar. Zonal averages derived from the data and models were also compared to demonstrate the validity of the modeled emission and the effects atmospheric seeing has on the inferred brightness temperatures (see Figure 6).

In summary, models of atmospheric temperatures and acetylene were constructed from retrievals that accounted for the specific viewing geometry. These models were used to produce synthetic images that simulated the original data at alternative viewing geometries, enabling us to compare thermal emission from the different epochs.

\section{Results}

\subsection{Upper Tropospheric Emission and Temperatures: 2018 versus 1986}

We compared observations and zonally averaged $18.7 \mu \mathrm{m}$ brightness temperatures from 2018 to 1986, as shown in Figure 7. The observed $18.7 \mu \mathrm{m}$ image and the synthetic image derived from the Voyager temperatures appear remarkably similar after accounting for random errors and the effects of diffraction and atmospheric seeing. The 2018 data appear only very slightly dimmer than the Voyager data everywhere except along the north edge of the planet. This can also be seen in the meridional plots of brightness temperatures derived from the images (Figure 7).

For the meridional plots, first the 2018 brightness temperatures were simply extracted directly from the observed 2018 image and the synthetic image that had been convolved with a PSF to mimic the effects of diffraction and atmospheric seeing suffered by our ground-based observations. By this comparison, the brightness temperatures in 2018 and 1986 are equivalent to within $0.2 \mathrm{~K}$ at most latitudes-roughly equal to the level of statistical uncertainty ( $3 \sigma$, estimated from the pixel-to-pixel standard deviation of the background sky divided by the square root of the number of pixels in our zonally averaged bins). Only along the southern flank of the equatorial maximum do differences slightly exceed these estimated 


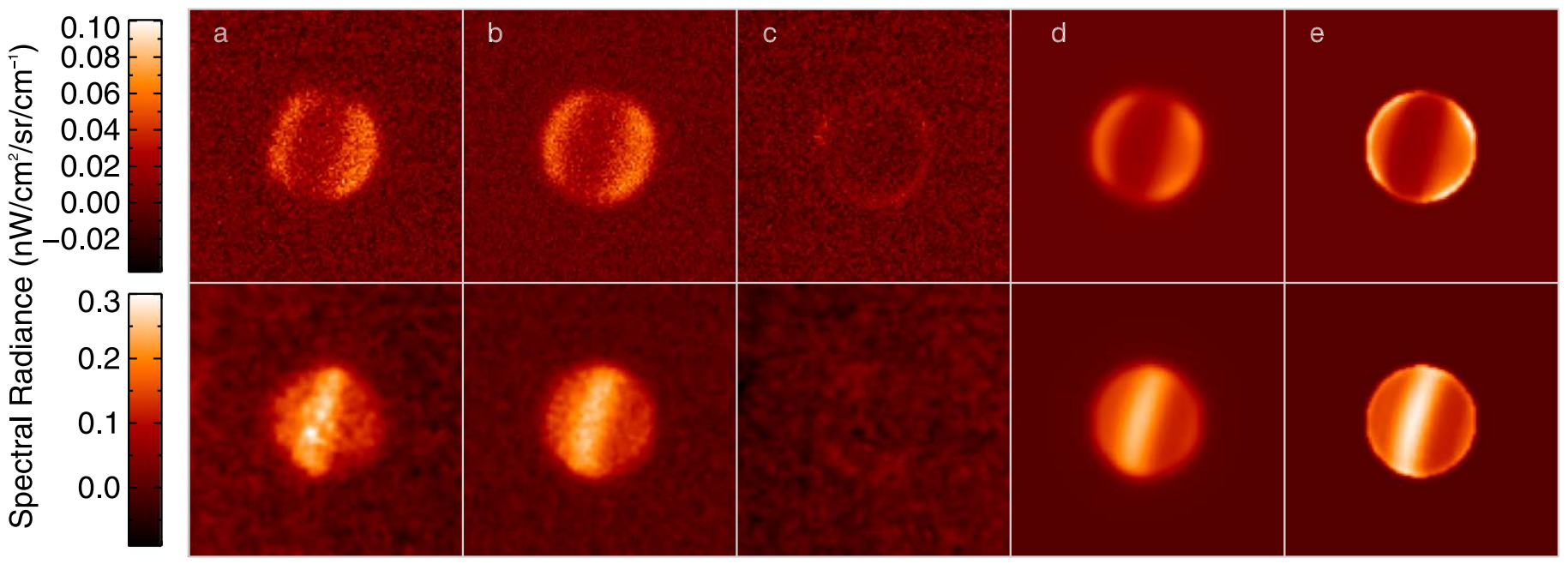

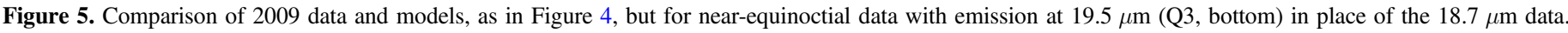
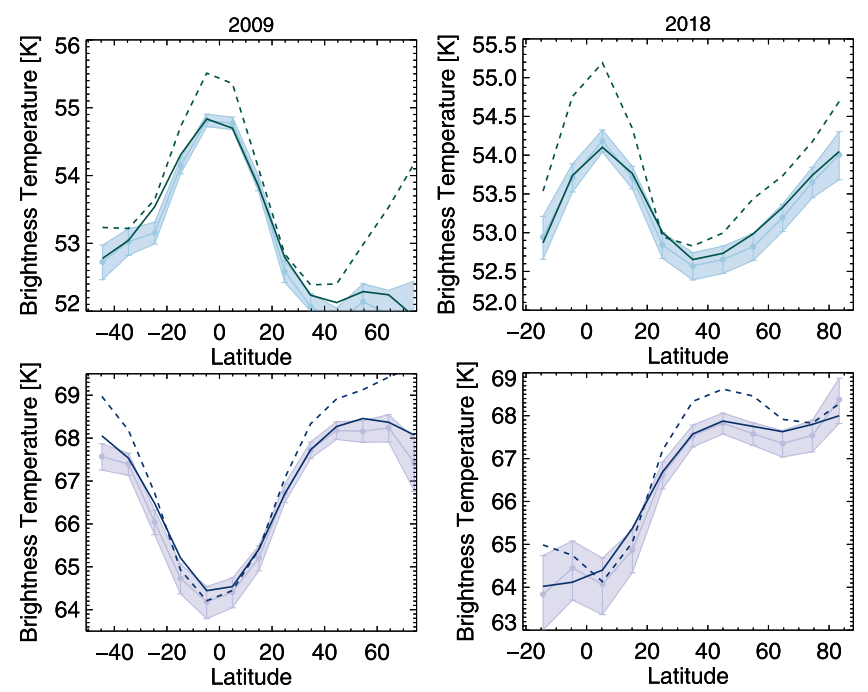

Figure 6. Comparison of models to the data from which they were derived. Curves depict zonally averaged brightness temperatures, binned $10^{\circ}$ in latitude, extracted from both observed and modeled images. The top two plots are for the tropospheric sensing filters: Q3 $(19.5 \mu \mathrm{m})$ in 2009 (left) and Q2 $(18.7 \mu \mathrm{m})$ in 2018 (right). The bottom two plots are for the stratospheric sensing filter, NeII_2 $(13.0 \mu \mathrm{m})$ in 2009 (left) and 2018 (right). The shaded regions represent the data with $3 \sigma$ uncertainties; the darker solid line represents the model convolved with a PSF to mimic the blurring suffered by the observations; and the darker dotted-dashed line depicts the brightness temperatures expected prior to convolution, representing an idealized model of intrinsic emission consistent with the observations.

uncertainties, but this sharp gradient is particularly sensitive to the image resolution and the modeled PSF.

To reduce potential differences due to imperfectly modeling the blurring, we additionally compared the 1986 brightness temperatures to equivalently blurred models of the 2018 data. These brightness temperatures were extracted from the blurred, modeled image derived from the 2018 retrieval (panel (d) in Figure 4 and the corresponding thick, solid curve in the upperright panel of Figure 6). In this case, the 1986 and 2018 brightness temperatures are remarkably similar, with a difference of less than $0.1 \mathrm{~K}$ at most latitudes. Subtle differences remain near the southern edge of the equatorial maximum and the north pole. In either case, the north pole is just over $0.2 \mathrm{~K}$ warmer in the 2018 data compared to the 1986 data. Some of this polar brightening may be attributed to a contribution from the rings, which appear near the pole but are not simulated in our data; however, from modeling, we estimate that this contribution would account for less than $10 \%$ of the observed difference.

Retrievals from the data suggest these small differences in brightness temperatures could be produced by atmospheric temperature near 100 mbar changing by $0.3 \pm 0.1 \mathrm{~K}$. However, given the limited information on the vertical profile from a single image, slight changes in the retrieved profile at poorly constrained heights can partly offset values at the peak of the contribution function, leading to retrieval uncertainties that are comparable to or larger than these changes. Even for the directly observed radiance, it is also important to note that we are comparing ground-based imaging to PSF-convolved forward models based on retrievals from spacecraft data, and so small systematic errors may remain despite our attempts to account for the observational differences. Considering the uncertainties, these results are consistent with changes of no more than $0.3 \mathrm{~K}$ in the brightness temperatures between 1986 and 2018, similar to what Orton et al. (2015) determined evaluating images dating from near equinox (ca. 2007), but with possible warming at the north pole at the limit of our uncertainty.

\subsection{Stratospheric Emission: 2018 versus 2009}

A comparison of zonally averaged brightness temperatures at $13 \mu \mathrm{m}$ from 2018 to 2009 images revealed a persistent peak in emission at northern midlatitudes with a minimum near the equator (Figures 6 and 8). The average 2009 distribution appears to be asymmetric with a minimum just south of the equator and a northern midlatitude peak in brightness temperature that is roughly $1.0 \pm 0.2 \mathrm{~K}$ warmer than the corresponding peak in the south (see Figure 8). We note that the 2009 data showed zonalmean variations in brightness temperatures of less than $0.5 \mathrm{~K}$ between the two consecutive nights, except at the equator where differences approach $1 \mathrm{~K}$ (Figure 8, left panel). These internightly differences are mostly consistent with the level of random noise in the images, but they may hint at coherent longitudinal variation (see Appendix $\mathrm{C}$ for more discussion on zonal variability).

A comparison between the 2009 and 2018 brightness temperatures extracted directly from the data at overlapping 

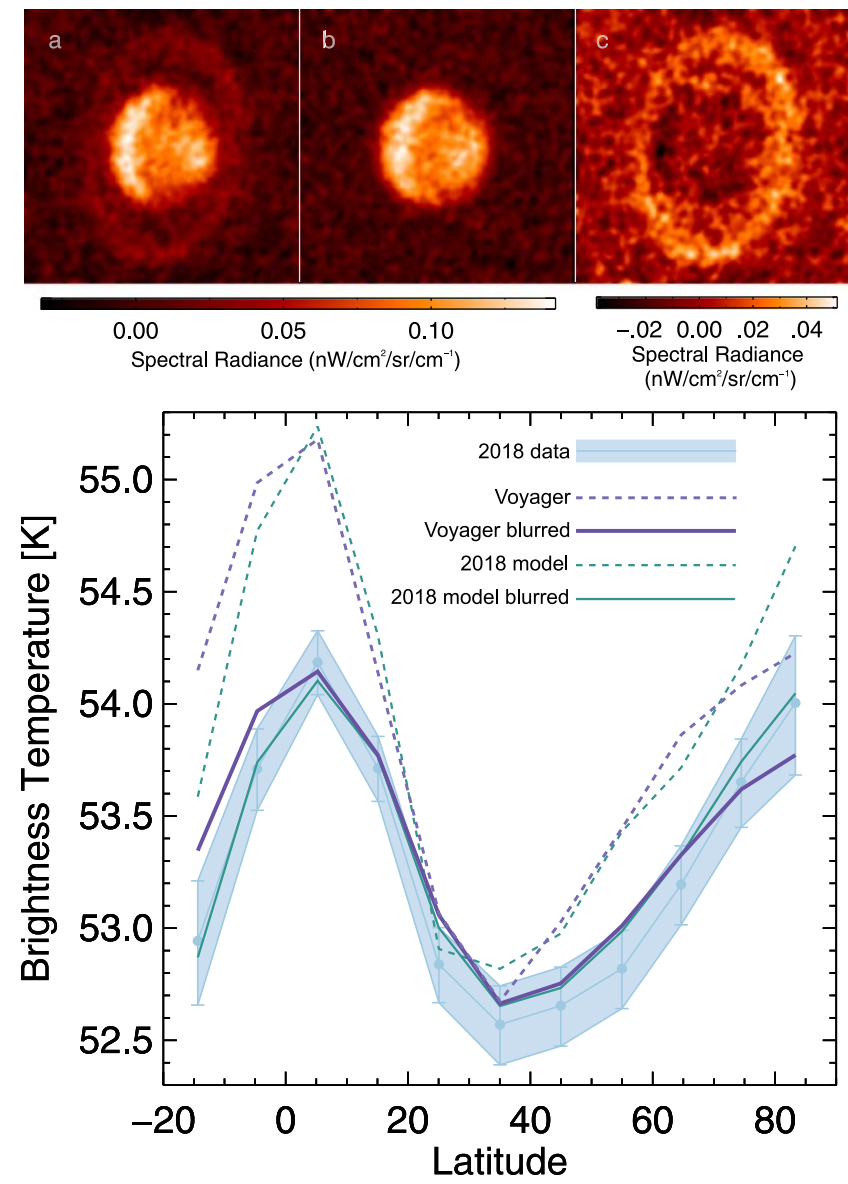

Figure 7. Comparison of emission in 2018 imaging data and 1986 Voyager data. (a) The $201818.7 \mu \mathrm{m}$ image; (b) a synthetic image created by forward modeling the temperatures derived from the 1986 Voyager/IRIS spectra (Hanel et al. 1986; Orton et al. 2015) to the same viewing geometry as the 2018 image. The model was convolved with a PSF, and synthetic noise was added for comparison. (c) The 2018 image minus the Voyager synthetic image with slight residuals limited to the northern pole, limb, and rings (which were not modeled in our synthetic images). (d) Zonally averaged brightness temperatures from the $18 \mu \mathrm{m}$ image (shaded) from 2018 compared to the equivalent brightness temperatures computed from the Voyager data. The light shading represents the statistical $3 \sigma$ uncertainty in the measurement centered around the mean value. The darker solid purple line shows the result of the forward-modeled Voyager emission convolved with the PSF for comparison to the ground-based data, while the dotted-dashed line depicts the Voyager brightness temperatures prior to being convolved with the PSF. Similarly, the modeled emission derived from the 2018 data (see Figures 4(d) and (e)) was also convolved with the PSF for comparison, shown as the solid dark green curve, along with the predicted emission prior to convolution (green dotted-dashed curve). The curves are remarkably similar, though the polar region in 2018 appears slightly brighter.

latitudes (up to emission angles of $78.5^{\circ}$ ) show agreement near the center of the disk, but display discrepancies at higher latitudes. Our modeling shows that these discrepancies can largely be explained by differences in the viewing geometries (see Figure 8, middle versus right panel). We showed this by essentially remapping our 2009 temperature model onto the 2018 viewing geometry (such that matching latitudes have identical emission angles) and forward modeling the expected emission (see Figure 9). Zonally averaged brightness temperatures drawn from this forward model show no significant change in brightness temperatures between $20^{\circ}$ and approximately $74^{\circ}$ north latitude. North of these latitudes, the temperature and acetylene were not actually retrieved in 2009 because of close proximity to the limb, and therefore our model of the 2009 atmosphere simply assumes an extension of the retrieved temperatures and acetylene at $\sim 74^{\circ}$. With this assumed atmospheric structure north of $\sim 74^{\circ}$, the forwardmodeled brightness temperatures are colder but still roughly consistent with the 2018 observations. However, south of $20^{\circ} \mathrm{N}$, we find that our modeled brightness temperatures are significantly greater than the observations. Due to the degeneracy in our retrievals, we find the same result regardless of we vary temperatures or acetylene in our 2009 retrievals.

If the southern hemisphere brightness temperatures from 2009 had persisted, our modeling indicates that we should expect to see a brighter southern limb in 2018, with blurring contributing to a brighter equator. As Figure 8 shows, this southern emission appears absent in the data. However, as Figure 9 shows, the difference between data and model demonstrates that this discrepancy exists along the entire limb and likely indicates a failure of the forward modeling of the limb rather than a physical change limited to the southern hemisphere. The modeled brightness along the limb is particularly sensitive to the assumed PSF (which convolves the disk and sky) and the vertical gradient in temperatures and acetylene, and so errors in either can produce modeling discrepancies. As we noted earlier, this can indicate that the upper stratospheric temperatures are increasing too rapidly in the adopted temperature model (Orton et al. 2014a) at pressures less than above 0.1 mbar. Temperatures (and acetylene) at these low pressures are not retrieved in our model because the contribution functions peak deeper in the atmosphere where the acetylene abundance is expected to be greater. Lower pressures are only sensed at the very edge of the disk, where even small amounts of uncorrected blurring between the planet and background sky can suppress observed radiances and lead to erroneously lower retrieved temperatures; hence, these locations (corresponding to emission angles beyond $\sim 72.5^{\circ}$, or $\mu<0.3$ ) were not included in our retrievals. Further investigations of the thermal center-to-limb behaviors and temperature lapse rates are beyond the scope of this paper but should be a goal of future work.

\subsection{Temperatures and Acetylene Derived from Stratospheric Emission}

To investigate sources of the stratospheric emission, we performed retrievals of temperatures and acetylene from the data. If stratospheric temperatures were allowed to vary (while acetylene was held fixed), we found the observed meridional pattern can be reproduced by a temperature gradient of roughly $13 \mathrm{~K}(13.4 \pm 2.8 \mathrm{~K}$ in 2018 and $12.4 \pm 2.8 \mathrm{~K}$ in 2009), measured at $0.25 \mathrm{mbar}$-roughly the pressure at which the $13 \mu \mathrm{m}$ contribution function peaks - from the near-equatorial minimum to the northern midlatitude peak at $\sim 40^{\circ}$ (see Figure 10). In the southern hemisphere, the peak temperature was roughly $4 \mathrm{~K}$ less. The variation in radiances was equally reproduced by a $500 \%-600 \%$ increase in the acetylene mixing ratio at northern midlatitudes $\left((2.7 \pm 0.7) \times 10^{-6}\right.$ volume mixing ratio $(\mathrm{VMR})$ increase in 2018 and $(2.0 \pm 0.6) \times 10^{-6}$ increase in 2009) relative to the expected values of Moses et al. (2018) and those retrieved at the equator. There was roughly $20 \%$ less at southern midlatitudes compared to the north, but these southern latitudes are only sampled in 2009 nearer the edge of the disk (see Figure 11).

While an increase in stratospheric acetylene had no effect on the inferred underlying upper tropospheric temperatures, 

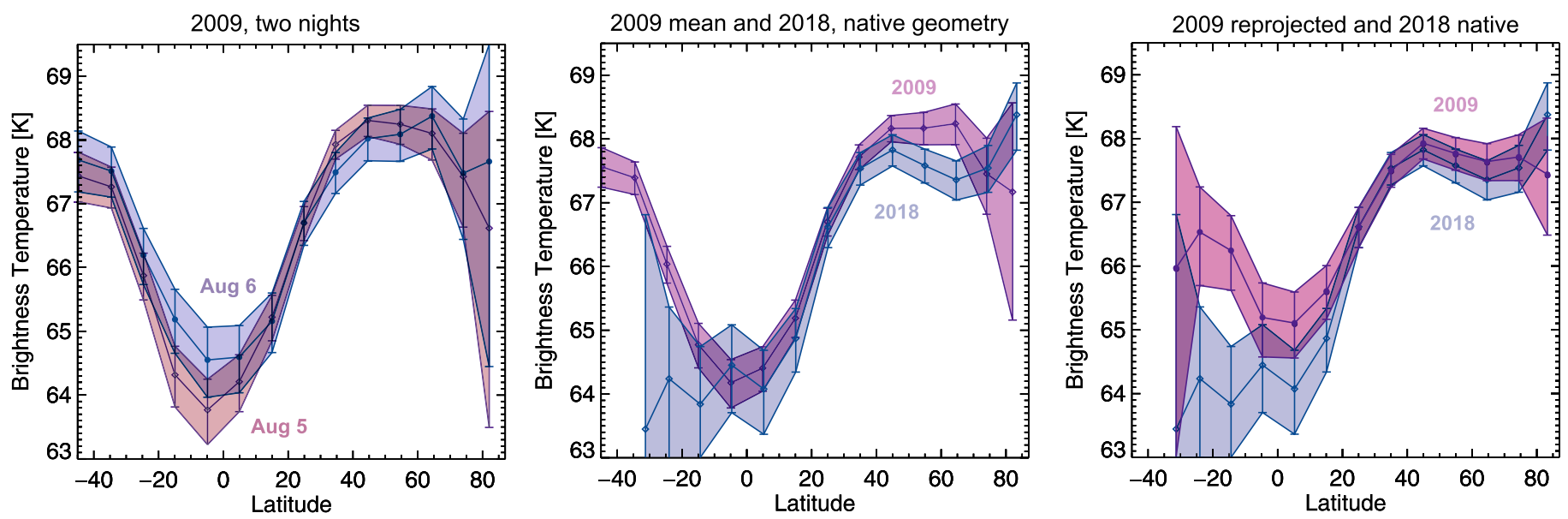

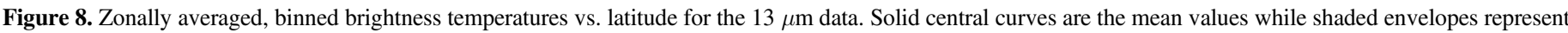

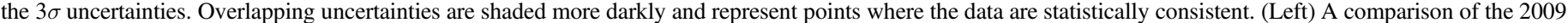

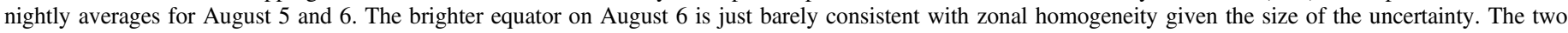

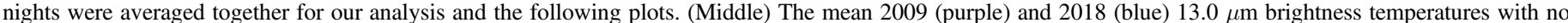

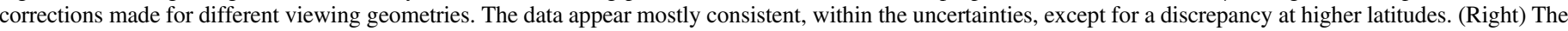

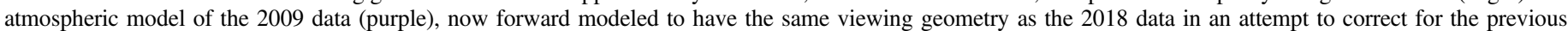

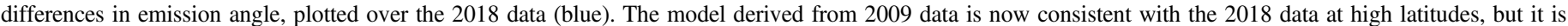

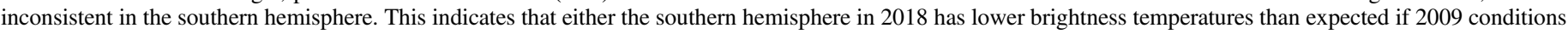
persisted, or that our modeling is strongly overcalculating the radiance toward the dimmer southern limb (see Figure 9).

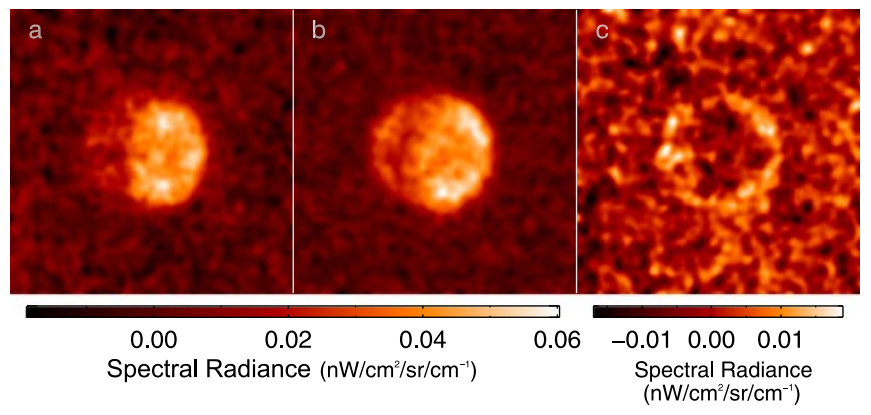

Figure 9. Comparison of observed and modeled emission at $13 \mu \mathrm{m}$ assuming the 2009 radiances persisted. (a) The 2018 image showing a dark southern limb to the left in the image. (b) The modeled emission assuming that the atmospheric temperatures or acetylene in 2009 had persisted until 2018. The synthetic image of expected emission in 2018 was produced by forward modeling the retrieved temperature field from the $200913 \mu \mathrm{m}$ data, though forward models created using the retrieved acetylene abundances (i.e., with temperatures held constant) appear identical. The forward-modeled image was convolved with a PSF and corrupted with synthetic noise for comparison. (c) The model minus the data shows that the model is consistently too bright along the limb, suggesting that much of the apparent brightening in the south may be due to an error in the temperature lapse rate assumed at the lowest pressures in our model and/or errors in the assumed PSF used to model atmospheric blurring.

we found that stratospheric warming would also be detectable at $18.7 \mu \mathrm{m}$ because of the broad contribution function of the Q2 filter. Therefore, we find that warming in the stratosphere must be compensated by cooling the troposphere to remain consistent with the observations. If the $13.0 \mu \mathrm{m}$ emission is due to increased stratospheric temperatures, then the inferred tropospheric temperatures may be up to $1 \mathrm{~K}$ colder at the midlatitudes relative to those inferred from the Voyager/IRIS data (assuming the longer wavelength Voyager measurements were not equally influenced by the contribution of warmer stratospheric temperatures). This difference is nearly comparable to the potential uncertainty in retrieved temperature profiles, but it would

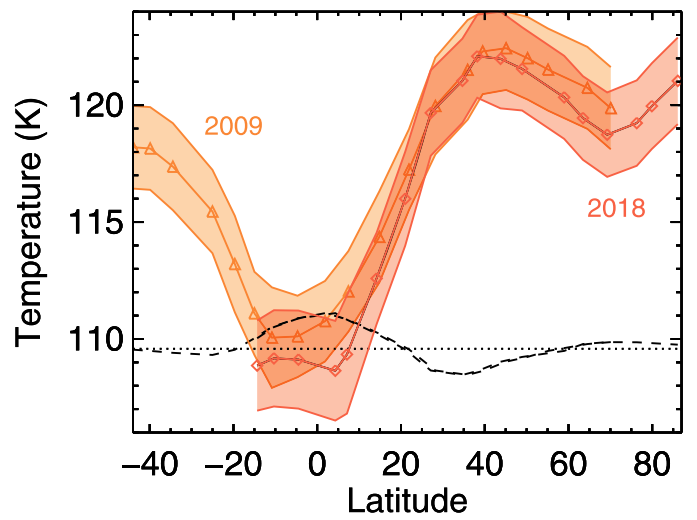

Figure 10. Retrieved atmospheric temperatures at $0.25 \mathrm{mbar}$-roughly the pressure of the peak contribution at $13 \mu \mathrm{m}$-consistent with the meridional variability in $13.0 \mu \mathrm{m}$ stratospheric emission assuming all variation is due to temperature change. The retrieved 2009 temperatures, shown with $3 \sigma$ uncertainties (triangles, lighter shading), are roughly consistent with the 2018 temperatures (squares, darker shading) given the uncertainties in the retrievals. The darkest shading indicates overlap between the two curves within the uncertainties. The dotted horizontal line marks the initial condition for the retrieval, while the dashed lines indicate the 0.25 -mbar temperatures from the model of Orton et al. (2015), which extends latitudinally resolved Voyager data to pressures less than 70 mbar by smoothly interpolating to the global $1 \mathrm{D}$ temperature profile of Orton et al. (2014a).

be consistent with cooling of the northern (autumnal) hemisphere following equinox.

\section{Discussion}

\subsection{Seasonal Changes}

The 1986 Voyager data reveal a meridional cross section of Uranus' temperatures near the tropopause shortly after the southern summer solstice. If we assume the atmospheric temperatures respond to solar heating and the radiative relaxation times approached a full season, the 1986 Voyager data would have displayed the atmosphere's thermal response 


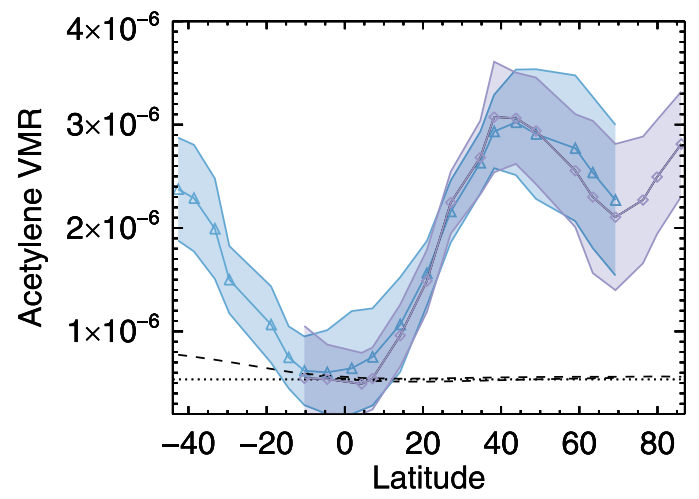

Figure 11. Retrieved acetylene volume mixing ratios (VMRs) at $0.25 \mathrm{mbar}$ consistent with the meridional variability of the stratospheric emission $(13.0 \mu \mathrm{m})$ assuming all variation is due to change in the acetylene abundance. The shading indicates $3 \sigma$ uncertainties in the VMR for data from 2009 (triangles, blue shading) and 2018 (squares, purple shading). The darkest shading indicates an overlap and a consistency between the two curves within the uncertainties. The dotted horizontal marks the initial condition for the retrieval, while the dashed lines indicate the 0.25 -mbar acetylene mole fraction from the model of Moses et al. (2018) for $2009\left(L_{s} \sim 7\right)$ and $2018\left(L_{s} \sim 46\right)$, nearly overlapping at mutual latitudes.

to solar forcing at the time of the preceding equinox in 1966 (Conrath et al. 1990). It then follows that the equinoctial data analyzed by Orton et al. (2015) would have provided a view of both hemispheres responding to asymmetric solstitial (southern summer, northern winter), and our 2018 observations would show the northern hemisphere's transition from winter to spring. The northern midlatitudes remained in darkness for nearly a decade past solstice in 1986, so it is plausible that cooling may have progressed beyond the 2007 equinox at these latitudes if the seasonal response lagged a full season behind the solar forcing.

Though uncertainties are significant, our data show that changes near the tropopause are consistent with atmospheric temperature changes no greater than $\sim 0.3 \mathrm{~K}$ since solstice, though perhaps slightly larger if potentially elevated stratospheric temperatures contributed to our retrieved temperatures (see Section 4.3). These values are consistent with the conclusion of Orton et al. (2015), who reported brightness temperature changes of less than $0.4 \mathrm{~K}$ between equinox and solstice.

The seasonal amplitude expected from radiative forcing will in general be reduced by a factor of $1 / \sqrt{1+r^{2}}$, where $r=2 \pi \tau_{R} / \tau_{O}$ and $\tau_{R} / \tau_{O}$ is the ratio of the radiative time constant to the orbital period (Conrath et al. 1998). Conrath et al. (1990) showed that a seasonal forcing of $10 \mathrm{~K}$ and a radiative time constant of $130 \mathrm{yr}$ should result in changes of $1 \mathrm{~K}$ or less. Using the updated, shorter time constants of Li et al. (2018), this value would be as large as $2-7 \mathrm{~K}$ with subseasonal lags (although this assumes the same $10 \mathrm{~K}$ forcing, which may not be consistent with the radiative heating applied by the authors). The amplitude and phasing derived from our data favor timescales of over $300 \mathrm{yr}$, which, considering the seasonal scales, amounts to essentially no seasonal variation due to solar forcing at midlatitudes. We emphasize that this is again based on seasonal radiative equilibrium temperatures from Conrath et al. (1990). Shorter radiative timescales would suffice if different heating rates and dynamical redistribution are assumed. Bezard \& Gautier (1986) computed seasonal contrasts of $4 \mathrm{~K}$ or less in the radiative equilibrium temperatures (depending on the latitude dependence of the internal heat flux), which would be consistent with radiative cooling times on order of a century. Shorter timescales computed by $\mathrm{Li}$ et al. (2018) would require equilibrium temperature swings of under $1.5 \mathrm{~K}$ or simply a greater amount of meridional mixing to overwhelm the radiative response (Friedson \& Ingersoll 1987). An updated radiativedynamical model using the latest absorption coefficients and chemical abundances is needed to better evaluate the consistency of these radiative time constants with the observations.

Higher in the stratosphere, our comparison is limited to a span of nine years. This span is short compared to estimates of long radiative and dynamical timescales on Uranus (Conrath et al. 1990; Moses et al. 2018), and so it is unsurprising to find little change in the northern hemispheric emission at $13 \mu \mathrm{m}$. By the same token, it would be surprising if any physical process could produce the apparent discrepancies between the 2018 data and 2009 model, further suggesting that this is likely attributable to an error in the assumed profiles of temperatures or acetylene abundances at higher altitudes that go into producing the forward model.

\subsection{Nature of the $13 \mu \mathrm{m}$ Emission}

The cause of the $13 \mu \mathrm{m}$ emission distribution is unknown, but as discussed in Section 4.3, it could result from a regionally elevated temperature, acetylene, or a combination of the two. Though not obvious from each pair of images alone, maps of the data show a remarkable anticorrelation between the 13 and $18.7 \mu \mathrm{m}$ radiances (see Figures 12(a), (b), and 13). Furthermore, while the midlatitudes are negatively correlated, both images show brightening at the poles. Together, these correlations suggest that a shared mechanism or mechanisms may be responsible for the emission in both filters, forming a dynamical link between stratosphere and upper troposphere.

One possible explanation for the observed emission may be elevated temperatures resulting from the adiabatic compression of regionally subsiding gas. Just as the meridional temperature structure in the upper troposphere has been interpreted as a consequence of large-scale vertical motions (Flasar et al. 1987), similar but separate circulation cells could possibly be at work higher in the stratosphere, rotating in an opposite sense to produce downwelling at midlatitudes and upwelling at the equator. The meridional position of this stacked circulation would not be merely coincidental, as it could be linked to the underlying meridional temperature gradient. While the driving force behind the upper tropospheric circulation is unknown (Flasar et al. 1987), the meridional temperature structure it produces has a maximum temperature gradient between $15^{\circ}$ and $20^{\circ}$ latitude in both hemispheres (with $d T / d y<0$ ). It is conceivable that this upper tropospheric temperature gradient could be geostrophically balanced by an unobserved stratospheric tropical jet aloft via the thermal wind relationship. Potential dissipative processes weakening the thermal wind with height could then lead to a mass-balancing meridional circulation with descending, warming air on the poleward side of the jet (Conrath \& Pirraglia 1983). Following Flasar et al. (1987; their Equation (3)), we can relate the desired steadystate temperature differences to a requisite differential vertical velocity by the expression

$$
\Delta w=\Delta T R / \tau_{R} H N^{2},
$$

where $\Delta T$ is the meridional temperature difference, $R$ is the gas constant $\left(\sim 3614.9 \mathrm{~J} \mathrm{~kg}^{-1} \mathrm{~K}^{-1}\right), H$ is the pressure scale height 

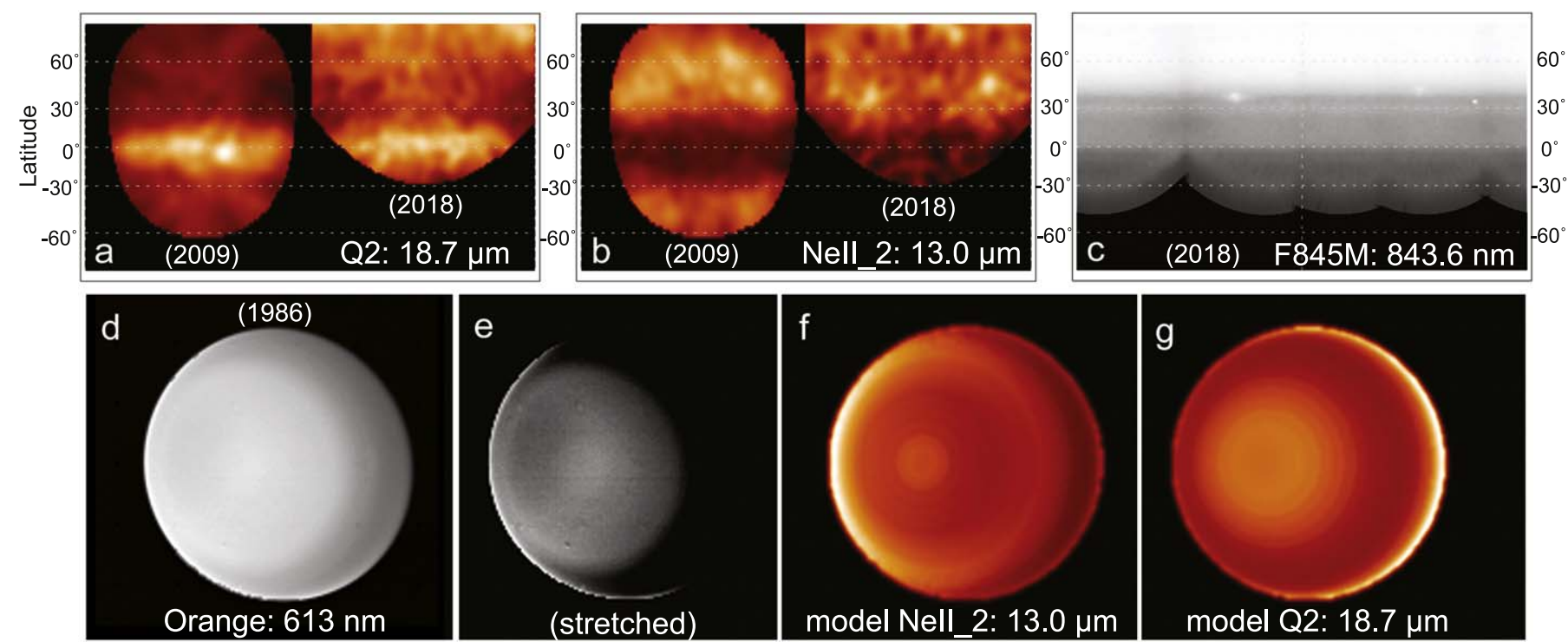

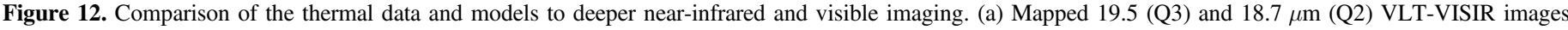

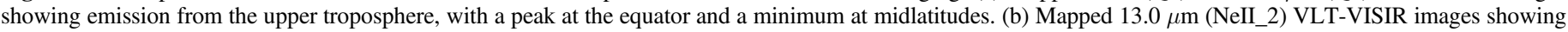

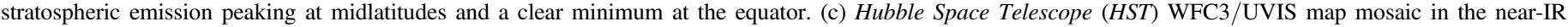

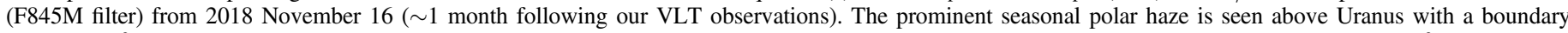

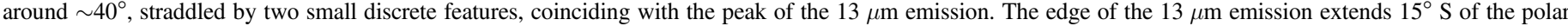

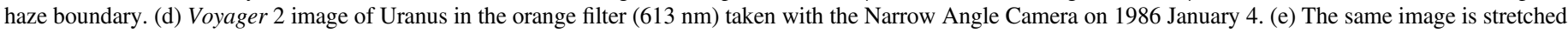

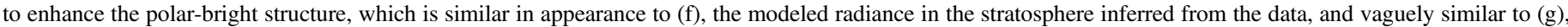

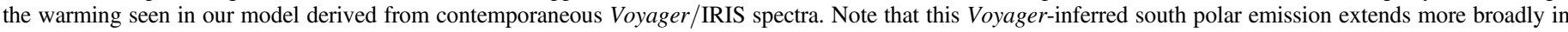
latitude compared to the equivalent north-polar emission retrieved from 2018 data (see Figure 4).
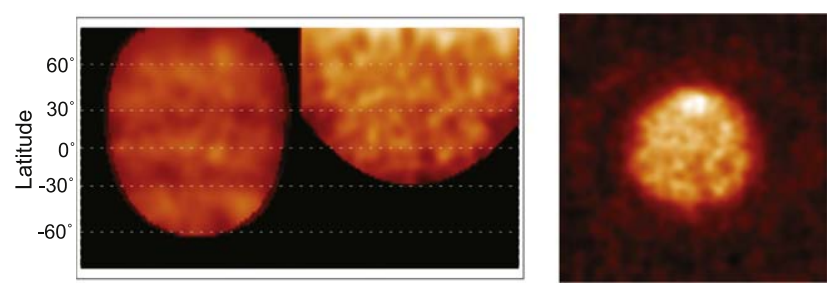

Figure 13. The combined sum of the 13 and scaled $18.7 \mu \mathrm{m}$ maps and images, where the anomalies essentially cancel each other out, illustrating the anticorrelation of radiances in the data at equatorial and midlatitudes. At the pole, the anomalies are both positive, leading to an enhanced bright spot. Together, these are consistent with upwelling at midlatitudes and downwelling at the pole. Radiances at $18.7 \mu \mathrm{m}$ were reduced by $50 \%$.

$(\sim 47 \mathrm{~km}), N$ is the buoyancy frequency $\left(0.0048 \mathrm{~s}^{-1}\right), \tau_{R}$ is the radiative time constant, and $(\Delta w)$ is the vertical velocity difference. Assuming $\tau_{R} \sim 49 \mathrm{yr}$ at $0.2 \mathrm{mbar}$ (Li et al. 2018) and $\Delta T \sim 13 \mathrm{~K}$ from our retrievals, we calculate $\Delta w \sim$ $2.8 \times 10^{-5} \mathrm{~m} \mathrm{~s}^{-1}$ - five times greater in magnitude than what Flasar et al. (1987) computed for the tropospheric vertical velocity differential.

While adiabatic warming associated with thermal winds could potentially explain the enhanced emission at midlatitudes, a different mechanism would be needed to explain the pattern in emission at higher latitudes. The apparent second peak in emission at the pole would be inconsistent with a thermal wind-driven circulation as the tropospheric temperature gradient is in the opposing direction at high latitudes (i.e., $d t /$ $d y>0$.) A separate mechanism such as wave heating or a larger, independent circulation would be needed to heat the pole. Ultimately, assessing the dynamical feasibility of this scheme requires evaluating the thermal wind equation and the corresponding mass conservation for zonal, meridional, and vertical components of the winds, which is beyond the scope of the present data and this paper.

Alternatively, a regional enhancement in acetylene could satisfy the $13 \mu \mathrm{m}$ emission with a much simpler and coherent dynamical circulation. If the putative midlatitude upper tropospheric upwelling simply extended farther into the stratosphere ( $\sim 6$ scale heights above the tropopause), tropospheric methane could potentially be mixed to lower pressures where it would photolyze to produce acetylene. As long as chemical conversion timescales were short compared to timescales for meridional transport, the concentration of acetylene would peak in the region of upwelling and diminish quickly to the north and south. Moses et al. (2018) suggest acetylene loss timescales are roughly $2 \mathrm{yr}$, with net lifetimes of $40 \mathrm{yr}$ at 0.2 mbar. Timescales of meridional transport are uncertain but potentially much larger, with Conrath et al. (1998) suggesting a dynamical timescale of $700 \mathrm{yr}$ in the stratosphere.

The resulting enhancement would be consistent with inferences of latitudinal variation in stratospheric hydrocarbons from the Voyager UVS data (Yelle et al. 1989; McMillan \& Strobel 1992). Yelle et al. (1989) reported a factor of 2-3 reduction in the observed reflectance in the 1338-1583 spectral band at midlatitudes relative to the pole and interpreted this signal as the relative depletion of hydrocarbons at the poles and enrichment due to extended upwelling at lower latitudes. For consistency, it then follows that the brightness detected at the poles at 13 and $18.7 \mu \mathrm{m}$ could both be potentially attributed to adiabatic warming in the sinking branch of this extended cell.

Regional enhancement of acetylene would also imply that methane would be similarly enhanced at midlatitudes. Though not detected in near-IR data (Karkoschka \& Tomasko 2009), regional enhancements in methane are indirectly consistent with inferences of supersaturated and spatially variable 
methane from Herschel PACS spectra (Lellouch et al. 2015), which found supersaturated methane mole fractions of $\sim 9.2 \times 10^{-5}$ at the tropopause-roughly six times larger than the value inferred from Spitzer (Orton et al. 2014b). The authors noted that the measurements could be reconciled if the methane profile decreased by a factor of 5 from 100 to 2 mbar, but such a profile would be difficult to explain. They argued that the discrepant supersaturated mole fraction may have instead indicated spatial heterogeneities in stratospheric methane abundance or temperatures, noting that the Herschel data probed more global conditions while the Spitzer data were biased toward warmer temperature regions. Interestingly, our data indicate a factor of 5-6 enrichment in acetylene in precisely the coldest regions (i.e., midlatitudes), while mole fractions at warm equatorial regions remain in strong agreement with values derived from Spitzer (Orton et al. 2014b; Moses et al. 2018), consistent with the proposed explanation for the observed discrepancy.

A vertically extended circulation cell has already been suggested for Neptune to explain correlations between observations in the stratosphere and troposphere (de Pater et al. 2014), and was previously proposed for Uranus by Yelle et al. (1989) and McMillan \& Strobel (1992). It is perhaps unsurprising that a similarly extended circulation may be found in the stratosphere on both planets, given their qualitatively similar, broad tropopauses (de Pater et al. 2014), despite the weaker vertical mixing and stronger seasonal forcing on Uranus. In either case, the upward flux of methane may have to exceed the presumed VMR limit imposed by the estimated equilibrium saturation vapor pressure at the tropopause (i.e., the cold trap). Evidence of potentially supersaturated mixing ratios of methane on Uranus and especially Neptune suggest that vertical mixing may indeed overcome the cold trap limitation (Lellouch et al. 2015), though the mechanisms of this process remain speculative (Lunine \& Hunten 1989; de Pater et al. 2014). Inversely, the greater vertical extent of the circulation cell would mean that upwelling gas would experience a greater and more extended flux of highenergy photons along its extended path compared to a circulation cell that ceased nearer the tropopause; this would serve to further deplete the gas of chemicals destroyed by photolysis, meaning only the most stable species would be present in the downwelling branches.

A strong coupling of the troposphere and stratosphere would also help to physically link the apparent hemispheric asymmetry seen in the tropospheric temperatures and $13 \mu \mathrm{m}$ emission. Both Voyager and equinoctial 13- $\mu \mathrm{m}$ imaging data show the equatorial maximum/minimum to be slightly offset to the southern hemisphere (Orton et al. 2015, 2018). Likewise, Voyager spectra and ground-based $18 \mu \mathrm{m}$ imaging showed the midlatitude temperature anomaly in the northern hemisphere to be roughly $1 \mathrm{~K}$ colder (i.e., a $\Delta T$ of $\sim 4$ versus $3 \mathrm{~K}$ ), while the $13 \mu \mathrm{m}$ emission from the northern hemisphere in 2009 appears roughly $1 \mathrm{~K}$ brighter than the southern hemisphere (a retrieved acetylene VMR of $3.0 \times 10^{-6}$ versus $2.3 \times 10^{-6}$ ). Both of these represent a roughly $30 \%$ difference that could be explained by upwelling that is greater in the north than in the south. Considering this asymmetry and the potential dynamical link, the apparent absence of emission along the southern limb in 2018 data may be an indication of an asymmetric extent of the upwelling, such that the acetylene mixing ratio and the contribution function peak at greater pressures and hence become less visible in the extended optical paths along the limb. Or perhaps the strength or position of the upwelling has changed in time (e.g., a slight southward shift of the southern hemisphere upwelling) due to deeper dynamical processes. Unfortunately, the southern hemisphere will soon be unobservable from Earth for several decades, so testing theories and confirming changes will have to remain a goal of future work and potentially visiting spacecraft.

In contrast to the elevated temperature scheme due to downwelling, upwelling would actually serve to reduce the temperature at midlatitudes through adiabatic cooling. Therefore, the alternative explanations of stratospheric downwelling and hydrocarbon upwelling would serve to produce temperature anomalies in opposite directions, although the cooling would be relatively minor due to the much weaker updrafts expected at these heights (Flasar et al. 1987). Independent observational constraints on the meridional temperature structure in the stratosphere can come from sensing the $\mathrm{H}_{2}$ $\mathrm{S}$ (1) quadrupole line and thus could help discriminate between these two theories. Trafton et al. (2012) note that 2007 Gemini TEXES observations show a bimodal but asymmetric pattern that is brightest toward the north, but further work is needed to determine the consistency with the above models.

One potential flaw with the extended upwelling mechanism is the apparent inconsistency at the pole and the equator. If downwelling produces adiabatic heating in the upper troposphere (i.e., at $18 \mu \mathrm{m}$ ) at the poles and equator, why does stratospheric downwelling appear to heat the stratospheric pole but not the equator? Even if the effect of the adiabatic heating was offset by the reduced acetylene, it is not obvious why the equator and pole should be different. Considering mass conservation, the considerably lesser surface area of the polar regions relative to the equatorial regions may naturally be a factor in producing greater adiabatic heating at the pole, but then we would also expect a similar effect in the deeper $18.7 \mu \mathrm{m}$ emission unless the area of subsidence expands with increasing depth. One possible solution is that the pole is also heated by independent dynamical mechanisms such as breaking waves or greater concentrations of absorbing aerosols, but this remains purely speculative. More detailed dynamical modeling will be necessary to help evaluate the circulation in the stratosphere, its link to the troposphere, and its overall changes in time.

\subsection{Comparison to Visual/Near-IR Imaging}

A comparison of the mid-infrared images to visible and nearIR imaging, dominated by absorption from methane and the scattering from clouds and hazes at greater pressures, shows some possible signs of correlations between the stratosphere and troposphere (Figure 12). Although the $13 \mu \mathrm{m}$ emission extends $\sim 15^{\circ}$ farther to the south, the boundary of the brighter polar northern haze layer in a near-IR Hubble Space Telescope (HST) image is at roughly the same latitude where the $13 \mu \mathrm{m}$ emission peaks $\left(\sim 40^{\circ}\right)$. Intriguingly, the bright discrete cloud features near $40^{\circ}$ in the $H S T$ image also roughly correspond in latitude to the brightest discrete signals seen in the nightly averaged $13 \mu \mathrm{m}$ images. The tenuous correlation might suggest that tropospheric vortices generate localized hydrocarbon enhancements through upwelling (de Pater et al. 2014) or localized heating via breaking waves, although a thorough analysis of the image noise suggests that these radiances would constitute noise at the $\sim 2.5 \sigma$ level and hence be inconclusive (see Appendix C). Establishing a link between upper tropospheric 
vortices and the stratosphere will require greater signal-to-noise imaging in future work.

If we assumed hemispheric symmetry in the meridional structure of the $13 \mu \mathrm{m}$ polar emission, we can compare our thermal model to images from Voyager. The polar aerosols seen from this perspective show an enhanced brightness very near to the pole in Voyager's orange $(590-640 \mathrm{~nm})$ and methane (614-624 nm) filters, with an extent that is similar to the pattern seen in our models derived from the stratospheric emission. The sharper increase at the pole is not evident in the Voyager/IRIS thermal spectra, which show a more gradual warming roughly coincident with the polar haze region. We note that we do see a similar sharper feature at the north pole in our $18.7 \mu \mathrm{m} 2018$ data (see Figure 4) even though it appears absent at the synthetic images generated from the Voyager spectra. If linked across the troposphere and stratosphere, these observations may indicate that stratospheric downwelling or photochemistry at the pole affects the albedo or abundance of the tropospheric haze layer $\sim 9$ scale heights below. This may potentially be accomplished by methane-depleted air settling from above, combined with photochemical products produced in the higher ultraviolet flux found in the stratosphere. Assuming the parent chemicals were derived from upwelling gases, the aerosols or particulates would have to be stable enough to survive meridional transport from midlatitudes to the pole and down to the cloud level, potentially over centuries (Conrath et al. 1998).

Considering the recent detection of hydrogen sulfide above the cloud layer (Irwin et al. 2018), a possible candidate for stratospheric aerosols may be stable sulfur allotropes, investigated as a possible source of haze in exoplanetary atmospheres (Zahnle et al. 2016; Gao et al. 2017). Trace amounts of upwelling $\mathrm{H}_{2} \mathrm{~S}$ could photodissociate in the stratosphere and combine with free $\mathrm{H}$ atoms (produced from the photolysis of $\mathrm{CH}_{4}$ ) to eventually form stable octasulfur allotropes (S8) capable of surviving further UV radiation and the range of temperatures found above the clouds. If carried with the circulation and concentrated at the pole before settling down upon the clouds, these yellow particulates (Meyer et al. 1972; Eckert \& Steudel 2003) could potentially explain the scattering preferentially seen in the orange $(614 \mathrm{~nm})$ filter relative to the blue (477 nm) and violet (431 nm) filters (Smith et al. 1989). It is, however, worth noting that this is purely speculative, and several other species of hydrocarbon polymers absorb in the violet and may potentially explain the visible spectra (Baines \& Bergstralh 1986; Pollack et al. 1986).

Polar aerosols could also potentially explain the slightly warmer summer temperatures at the poles in the lower stratosphere seen in the Voyager data (Orton et al. 2015). If this were due to solar heating, such changes would only be expected from the radiative-dynamical model of Conrath et al. (1990) if radiative time constants were significantly shorter than even those of Li et al. (2018) at stratospheric heights. This could potentially be caused by unaccounted presence of absorbing aerosols. Likewise, the broader polar brightness seen in the model $18.7 \mu \mathrm{m}$ emission derived from the Voyager IRIS spectra may indicate broader subsidence or additional heating associated with overlying hazes or deeper tropospheric aerosols seen in the Voyager imaging. However, in terms of seasonal changes, the vertical velocities implied by the temperature variations $\left(\sim 10^{-5} \mathrm{~m} \mathrm{~s}^{-1}\right)$ are still comparable to or smaller than the expected fall velocities of photochemical aerosols (Toledo et al. 2019). Therefore, if estimates of settling times and vertical velocities are correct, downwelling may not significantly aid the transport of material from the stratosphere to the cloud level on timescales short enough to explain seasonal changes in the appearance of polar regions. Therefore, any apparent correlations in structure may be signs of the mean annual signatures of transport imprinted on the seasonally varying albedo of the haze layer, itself controlled by mechanisms that have yet to be fully explained. If seasonal changes are related to temporal changes in the methane mixing ratio above the cloud layer, it is conceivable that this process may be aided or triggered by a seasonal increase in the rate of subsidence. If true, this process may be detectable by measuring changes in the polar thermal emission, although as our data show, it is challenging to measure the polar regions near equinox given the geometry seen from Earth.

If variations in the seasonal albedo are related to changes in temperatures-whether through changes in condensation, subsidence, or convective stability - these temperature changes are not apparent in our upper tropospheric or stratospheric data. Li et al. (2018) computed radiative time constants as short as a decade near the polar cloud tops (Sromovsky et al. 2019), but the variation of cloud layer temperatures have yet to be measured. We have shown that radiative time constants at higher altitudes are either longer than expected or mixing can effectively dampen seasonal temperature changes. If meridional mixing is present in the variable cloud layer as well, it apparently does not traverse the boundary at $40^{\circ}$ latitude.

\section{Conclusions}

Analysis of our ground-based mid-infrared imaging of Uranus by VLT/VISIR in 2018 has revealed persistent thermal structures of the troposphere and new insight into the circulations of the stratosphere.

Uranus' upper tropospheric temperatures have changed little since the 1986 solstice, with the basic structure consistent with persistent midlatitude upwelling (Flasar et al. 1987). Brightness temperatures measured from zonally averaged meridional profiles are consistent with changes of less than $0.3 \mathrm{~K}$ in the $32 \mathrm{yr}$ since the southern summer solstice, with possible warming at the north pole due to adiabatic compression, aerosol heating, or other dynamical processes. These small changes are consistent with extremely long radiative timescales or very efficient meridional heat transport, suggesting the need for updated radiative-dynamical models.

To evaluate potential changes in the stratosphere, we compared imaging data at $13 \mu \mathrm{m}$ from 2018 to 2009 and found little change in the northern midlatitudes. We find a significant asymmetry between the northern and southern midlatitudes in 2009 images that may be associated with an asymmetry in dynamical mixing or unexpected changes in the photochemistry. Though constraining observations of the southern hemisphere may not be available again until the southern hemisphere returns into view in the late 2030s, modeling should aim to test potential explanations for the hemispheric asymmetry.

The meridional structure of the stratospheric emission nearer the pole came into view in 2018 , allowing us to infer a possible peak at midlatitudes that appears remarkably anticorrelated with the $18 \mu \mathrm{m}$ emission from deeper in the atmosphere. The $13 \mu \mathrm{m}$ midlatitude peak also shows possible longitudinal structure that roughly coincides with the bright cloud features and a transition in reflectance within the cloud layer below. We 

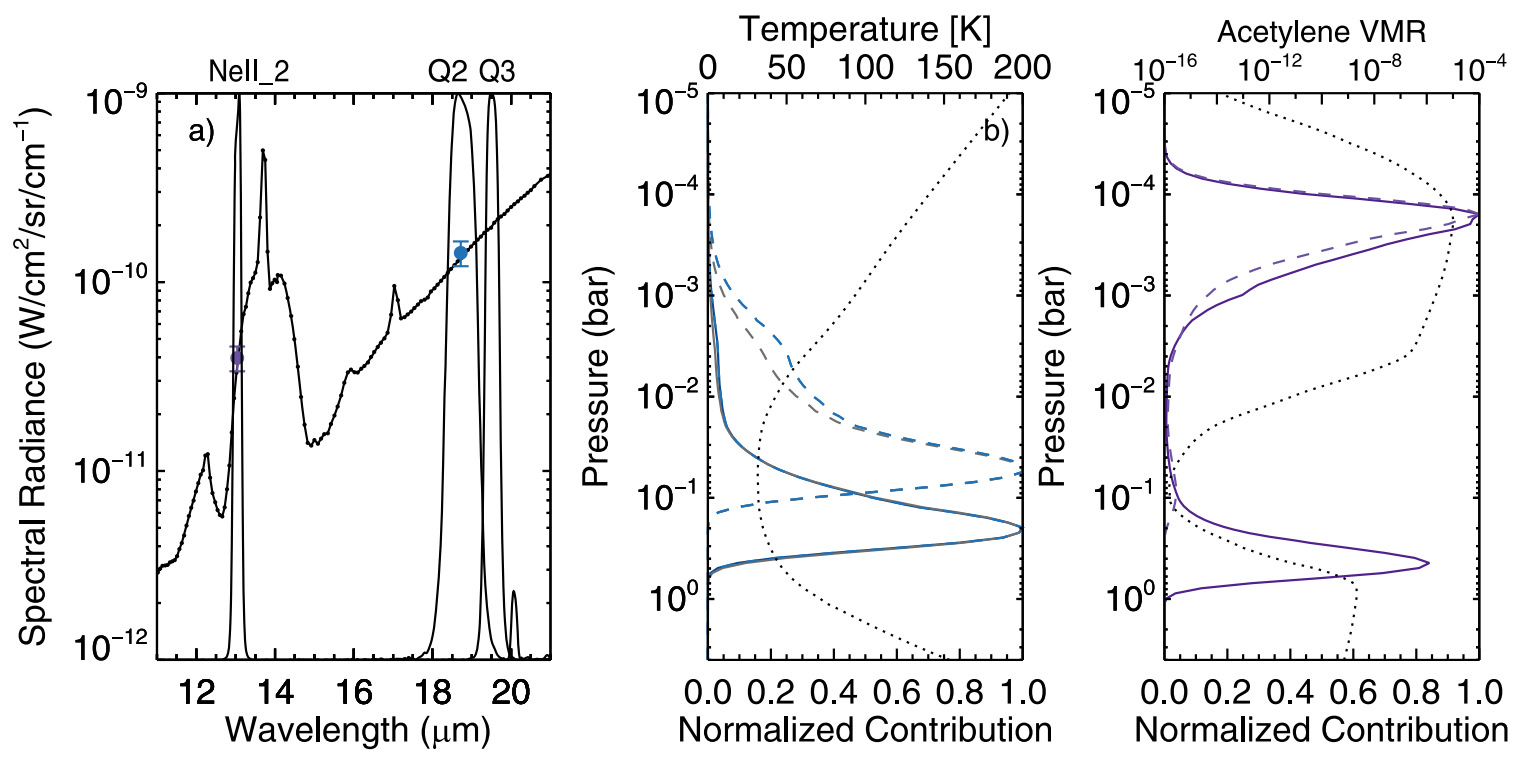

Figure 14. (a) 13.04 (NeII_2), 18.72 (Q2), and $19.50 \mu \mathrm{m}$ (Q3) normalized filter transmissions superimposed over the Short-Low Spitzer spectra of Orton et al. (2014a). The NeII_2 filter senses emission from acetylene while the broader Q2 and Q3 continuum filters measure atmospheric temperatures via collision-induced hydrogen emission. Disk-integrated values of the 2018 measurements are plotted with error bars representing a $20 \%$ uncertainty. (b) The normalized contributions for each filter superimposed at nadir (solid line) and limb (dashed). The middle panel shows the 18.72 (blue) and $19.50 \mu \mathrm{m}$ (gray) contribution functions along with the temperature profile (dotted) of Orton et al. (2014a) shown for comparison. These $Q$-band filters probe the upper troposphere and tropopause, with the lowest pressures toward the limb. The right panel shows that contribution to the $13.04 \mu \mathrm{m}$ (NeII_2) emission peaks in stratosphere at all emission angles, although there is a significant contribution from the upper tropopause near nadir. The dotted line in this panel plots the globally averaged acetylene VMR at equinox from Orton et al. (2014a) and Moses et al. (2018).

interpret these correlations as indicators of a potential link between the stratosphere and troposphere. Although the stratospheric emission may be due to regionally warmer temperatures produced by downwelling, we believe an enhancement of a factor of roughly 5 in the acetylene mole fraction at midlatitudes can explain the observations more simply. This implies that the upper tropospheric circulation pattern inferred from Voyager data (Flasar et al. 1987) extends at least six scale heights into the stratosphere and is capable of transporting hydrocarbons higher than previously appreciated. Thus, we interpret the observed pattern of acetylene as possible evidence that methane is primarily transported from the troposphere, through the cold trap, and into the stratosphere where it is subsequently photolyzed into acetylene and limited to midlatitudes. This would suggest a commensurate enhancement of methane near the tropopause at midlatitudes, potentially consistent with supersaturated or spatially variable methane profiles inferred from Herschel (Lellouch et al. 2015). We argue that the long path through the stratosphere along this extended circulation cell leads to greater photolytic depletion of hydrocarbons in the corresponding downwelling branches expected at the equator and poles, accompanied by adiabatic warming that extends from the middle stratosphere to at least the upper troposphere, particularly at the poles.

Our analysis is based on a limited number of noisy images and much of what we discussed is speculative, given uncertainties in the data and a lack of published dynamical models. The unprecedented sensitivity of the James Webb Space Telescope promises to greatly improve our understanding by providing unambiguous characterization of the thermal structure of the north pole in the 2020s. Dynamical models of Uranus' uniquely forced atmosphere and obscure circulations will be needed to help interpret present observations and the many unexpected findings to come.
This analysis was based on observations collected at the European Organization for Astronomical Research in the Southern Hemisphere under ESO programmes 0101.C-0073(B) and 083.C-0162(A). This work was supported by a European Research Council Consolidator grant, under the European Unions Horizons 2020 research and innovation programme, grant No. 723890. L.N.F. was also supported by a Royal Society Research fellowship. G.S.O. was supported by NASA through funds distributed to the Jet Propulsion Laboratory, California Institute of Technology. This work used the ALICE supercomputing facilities provided by the university of Leicester. This work used data acquired from the NASA/ESA HST Space Telescope, associated with OPAL program (PI: Simon, GO13937), and archived by the Space Telescope Science Institute, which is operated by the Association of Universities for Research in Astronomy, Inc., under NASA contract NAS 5-26555. All maps are available at https://doi.org/10.17909/T9G593.

\section{Appendix A}

Filter Transmissions and Contributions

Normalized transmissions and atmospheric contributions for the NeII_2 and Q2 filters are shown in Figure 14. Filter transmissions were taken from the ESO's VISIR instrument webpage. ${ }^{4}$ The emission observed through these filters is attributed to a range of atmospheric pressures dependent upon the atmospheric temperature, density, and opacity. These atmospheric contribution functions were evaluated for each filter as the functional derivatives of radiance with respect to temperature using the NEMESIS radiative-transfer suite (Irwin et al. 2008) and the atmospheric model of Orton et al. (2014a).

\footnotetext{
4 https://www.eso.org/sci/facilities/paranal/instruments/visir/inst.html
} 


\section{Appendix B Correcting for Observational Effects}

The observed radiance for each point on the planet's disk generally depends on the local emission angle $(\mu)$ as determined by the time-dependent viewing geometry as seen from Earth. Consequently, differences in the observed emission angle alone lead to apparent changes in the observed radiance, and a simple, direct comparison of the new and archival images was hindered by changes in the observing geometry over the intervening nine years. In addition, imperfect seeing and optical diffraction significantly reduced the observed radiance nearer to the limb of Uranus' relatively small disk, altering the observed center-to-limb variation in emission. If this effect was not correctly accounted for, the retrievals from images would have yielded erroneously colder temperatures near the limb in our model of the true atmosphere (prior to diffraction and atmospheric distortion). In theory, this blurring could have been corrected for by performing a mathematical deconvolution between the image and the effective PSF; however, in practice, the process amplified the considerable noise in our images, overwhelming the signal in the absence of excessive smoothing, rendering the deconvolution profitless.

In order to develop an accurate model of latitudinal temperature structure prior to blurring, we attempted to account for losses near the limb by first evaluating the effect of observed PSF on synthetic images and then using these results to create simple correction factors. This was done as follows: First, we extracted zonal averages of the radiances from the filtered images, avoiding points near the edge of the disk $(\mu<0.3)$. These zonally averaged radiances were then inverted to retrieve atmospheric temperatures or acetylene using an optimal estimation retrieval algorithm (NEMESIS; Irwin et al. 2008). Beginning with initial profiles of atmospheric temperatures and acetylene based on Orton et al. (2014a), the inversion yielded optimized continuous profiles of atmospheric parameters as a function of pressure and latitude. For the latitudes corresponding to omitted points near the edge of the disk (i.e., points with emission angles greater than $72.5^{\circ}$ ), values at the nearest sampled latitudes were used. We then mapped these parameter profiles onto a disk (assuming zonal homogeneity) and computed the emerging radiances using NEMESIS, resulting in a synthetic image of the planet for each filter. These synthetic images were convolved with appropriate PSFs (carefully determined from the corresponding stellar images) to yield synthetic blurred images. By dividing the original synthetic images by the blurred synthetic images, we obtained simple, twodimensional factors that approximated how the convolutions altered the images. We then applied these correction factors to the real data to approximately reconstruct the true center-to-limb variation in each image, before once again extracting zonal averaged radiances and retrieving an improved temperature structure. Though this approach was not a technical deconvolution, we found that it adequately mimicked the general effect and ultimately allowed us to generate more accurate models of the observations. As noted, however, the limb brightening near the very edge of the disk was too great in our modeling, indicating a need for improvement in future modeling.

Modeling the images allowed us to characterize the different components that ultimately led to the final image (i.e., the signal, noise, diffraction, atmospheric blurring, viewing geometry) while yielding temperature and acetylene models that could be analyzed and remapped to different times and geometries. This approach also had the benefit of allowing us to compare and adapt models to the unaltered observations without the risk of corrupting the true data themselves by correcting for observational effects. The drawbacks are that the models are sensitive to assumptions regarding vertical profiles that cannot be constrained by our data. The anomalous limb brightening discussed in the text is likely a consequence of our assumed profile; however, in its failure, this modeling provides motivation for correcting the temperature profile in future work.

\section{Appendix C \\ Hints of Zonal Variability}

All of the zonal variations evident in the individual 2018 images are consistent with image noise, as seen by comparing the actual data to the modeled maps using realistic noise (computed from the standard deviation of the background skies). This is true for the averaged images as well as the individual images. For example, Figure 15 shows the mapped
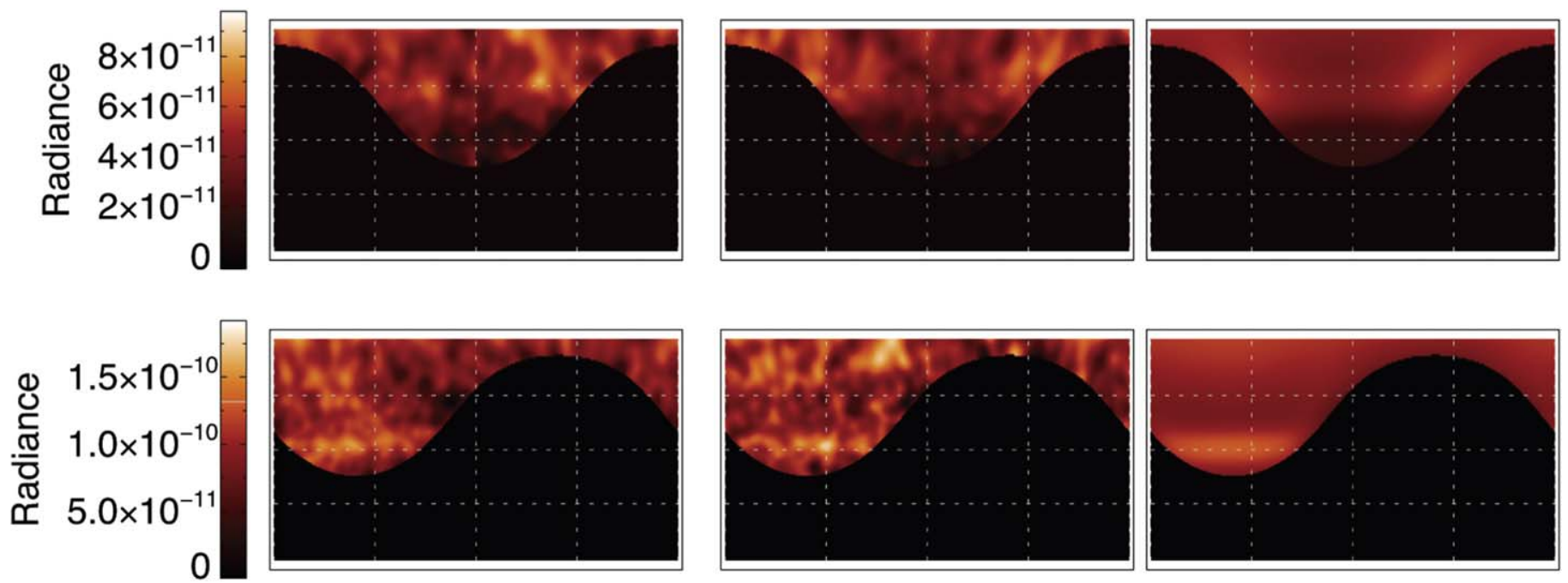

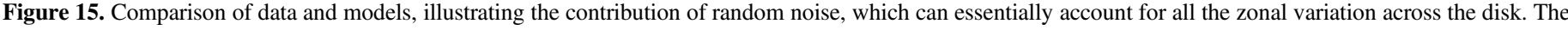

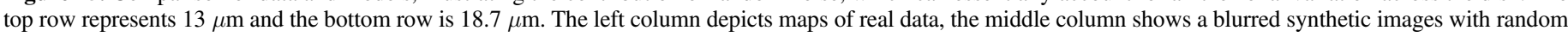

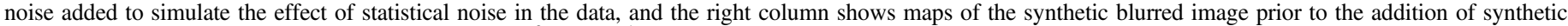
noise. Radiances in the color bar are in units of $\mathrm{W} / \mathrm{cm}^{2} / \mathrm{sr} / \mathrm{cm}^{-1}$. 


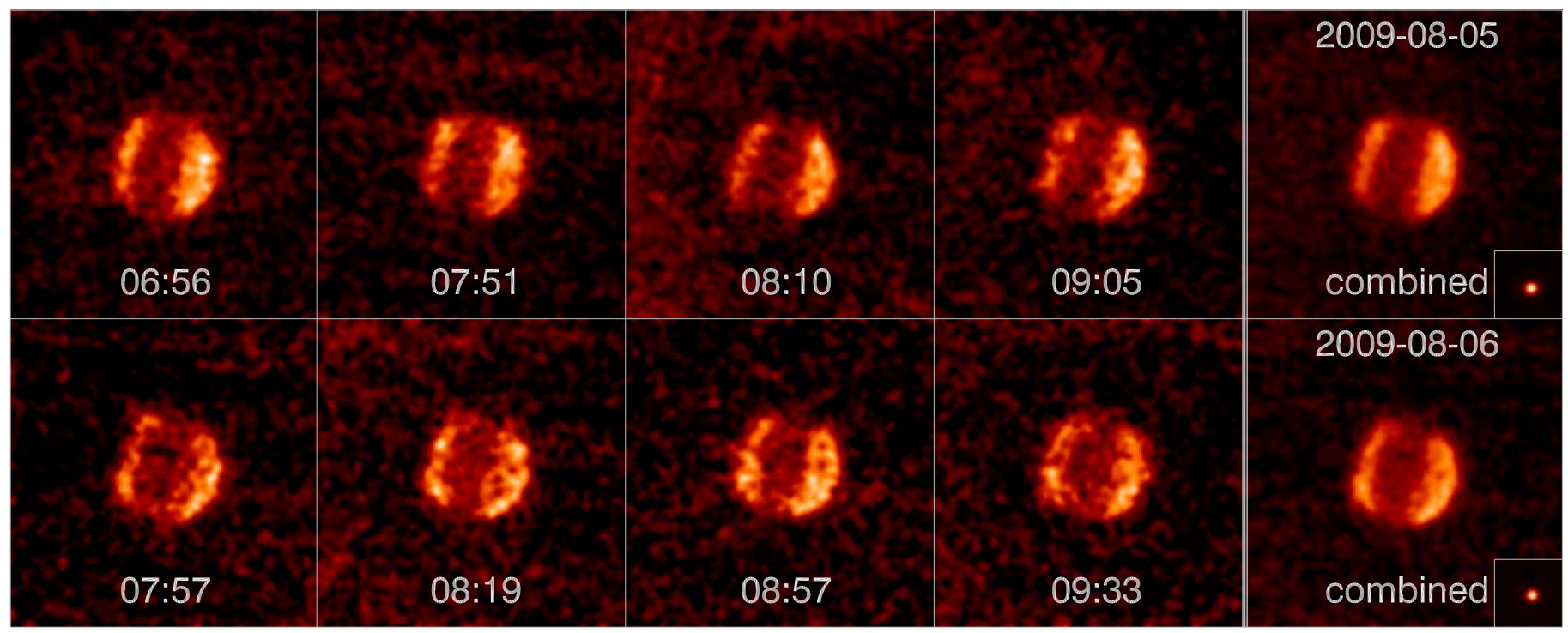

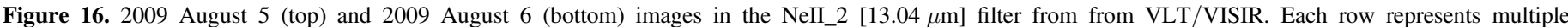

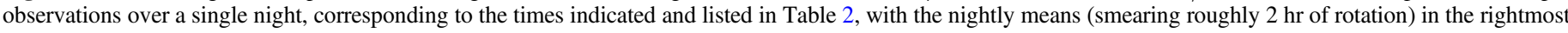

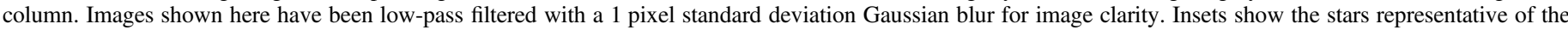

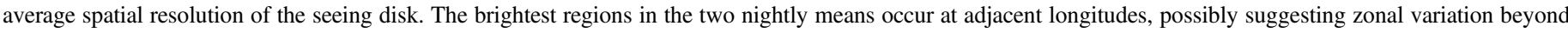
the noise. For our analysis, we chose to average both nights together (see Figure 2).

individual images alongside synthetic maps created from our model. This justifies the choice to average individual images together given the low $\mathrm{S} / \mathrm{N}$. However, it is worth noting the brightest features in the NeII_2 intriguingly appear at similar latitudes $\left(\sim 40^{\circ}-45^{\circ}\right)$ and could constitute real features at the $\sim 2.5 \sigma$ level. The $200913 \mu \mathrm{m}$ images also reveal hints of possible zonal variability with coherently greater emission toward similar longitudes (as shown in Figure 16), but these are marginally consistent with the level of distortion due to image noise and blurring. Zonal variability in the $13 \mu \mathrm{m}$ emission would help explain variations between different longitudes noted in the disk-averaged Spitzer observations (Orton et al. 2014a). Investigating possible discrete physical features and zonal variability in the stratosphere will require greater $\mathrm{S} / \mathrm{N}$ imaging in the future.

\section{Appendix D \\ Variations in Time due to Changes in Observing Geometry}

Finally, regardless of the cause of the seasonal cloud variability, decades of disk-integrated visible albedos (472 and $551 \mathrm{~nm}$ ) show an asymmetric seasonal pattern that cannot be explained by viewing geometry alone (Lockwood 2019). Although these historical measurements do not yet cover a complete cycle to evaluate changes in solstices, they do show a clear asymmetry between equinoxes. The 1946 southern vernal equinox has a significantly lower albedo than the 2007 northern vernal equinox, reaching seasonal minimum values shortly after each equinox. We can attempt to compare this pattern of observed visible albedo to the expected variation in the thermal emission.

Combining retrieved temperatures from Voyager (Orton et al. 2015) with 2009 and 2018 temperatures retrieved from imaging, we developed a global model of temperatures. This was then forward modeled over a range of viewing geometries encompassing a full orbit of Uranus as seen from Earth (see Figure 17). The model assumed that the asymmetry in 2009 imaging was invariant. The variations we see are thus asymmetric in time, although the south polar regions are purely speculative and assumed to be symmetric with the north at $13 \mu \mathrm{m}$ and consistent with Voyager at $18 \mu \mathrm{m}$. However, the vernal and autumnal equinoxes are symmetric, unlike what is seen in the visible (Lockwood 2019), so these thermal curves provide no obvious clues to the unexplained visible asymmetry.

Given discrepancies between the data and photochemical model, we ignored seasonal variations in the photochemical abundances (Moses et al. 2018), opting instead to use the retrieved abundances held fixed over the year.

From a practical observational perspective, the range in brightness temperatures is subtle and probably undetectable in the published record of observations given larger uncertainties in calibration at these wavelengths, which may be as large as $30 \%$ in radiance. For comparison, Orton et al. (1987) measured Uranus brightness temperatures in 1985 of $\sim 63 \mathrm{~K}$ at $13 \mu \mathrm{m}$ and just greater than $53 \mathrm{~K}$ at $18.7 \mu \mathrm{m}$, both of which fall below the plotted ranges of our annual projections. 

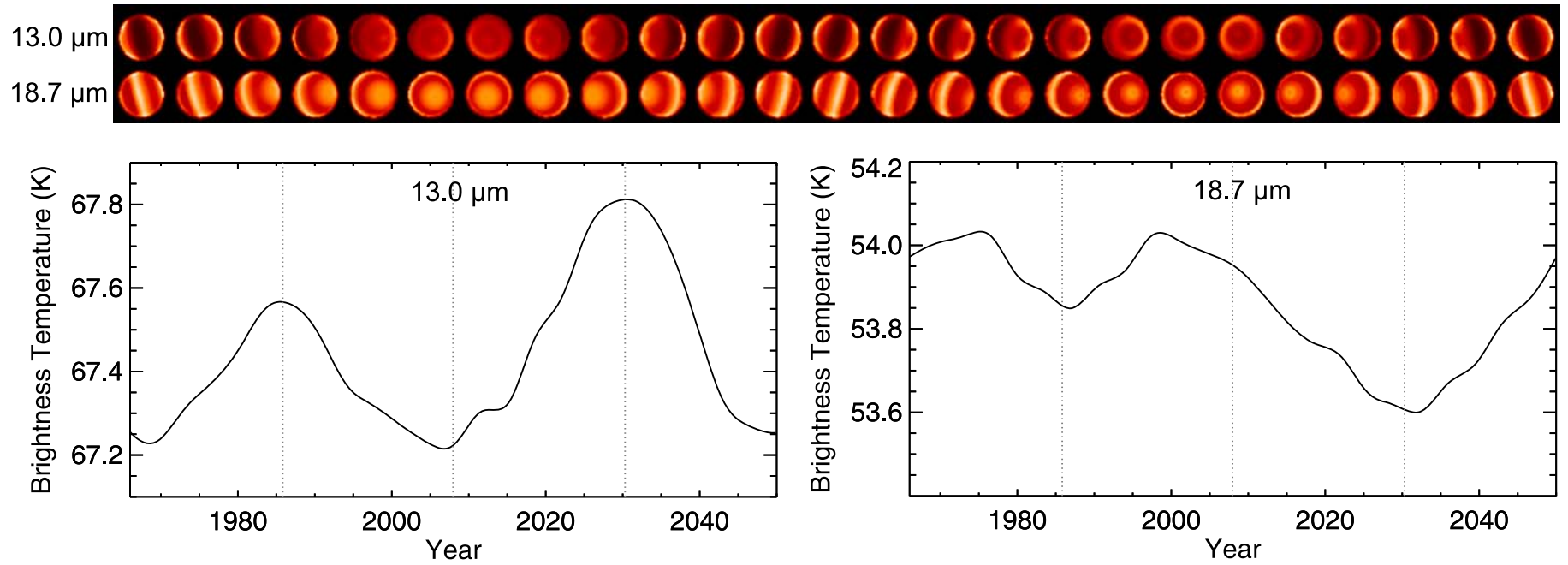

Figure 17. Variations in the radiance due to differences in observing geometry between the 1966 and 2050 equinoxes at intervals of 3.5 yr. (top) Synthetic images of thermal emission at 13 (top row) and $18.7 \mu \mathrm{m}$ (bottom row) produced by forward modeling our hybrid aggregate model of temperatures from 2018, 2009, and Voyager. Images show the view from Earth at $3.5 \mathrm{yr}$ intervals with the disk size normalized to correct for secular variations from Earth's orbit; these secular variations were accounted for in our disk-integrated brightness. (bottom) Disk-integrated brightness temperatures for 13 (left) and $18.7 \mu \mathrm{m}$ (right) produced from a spline fit to the integrated synthetic images. The assumed asymmetry is evident, but variations are still small relative to typical calibration uncertainties.

\section{ORCID iDs}

Michael T. Roman (1D https://orcid.org/0000-0001-8206-2165 Leigh N. Fletcher (iD https://orcid.org/0000-0001-5834-9588 Glenn S. Orton (iD https://orcid.org/0000-0001-7871-2823 Naomi Rowe-Gurney (iD https://orcid.org/0000-0001-8692-5538 Patrick G. J. Irwin (iD https://orcid.org/0000-0002-6772-384X

\section{References}

Baines, K. H., \& Bergstralh, J. T. 1986, Icar, 65, 406

Bezard, B., \& Gautier, D. 1986, in The Jovian Atmospheres, ed. M. Allison \& L. D. Travis (New York: NASA Goddard Space Flight CenterInstitute for Space Studies), 254

Conrath, B., \& Pirraglia, J. 1983, Icar, 53, 286

Conrath, B. J., Gierasch, P. J., \& Leroy, S. S. 1990, Icar, 83, 255

Conrath, B. J., Gierasch, P. J., \& Ustinov, E. A. 1998, Icar, 135, 501

de Pater, I., Fletcher, L. N., Luszcz-Cook, S., et al. 2014, Icar, 237, 211

Dobrzycka, D., \& Vanzi, L. 2008, in The 2007 ESO Instrument Calibration Workshop, ed. A. Kaufer \& F. Kerber (Berlin: Springer), 139

Eckert, B., \& Steudel, R. 2003, in Elemental Sulfur und Sulfur-Rich Compounds II, ed. R. Steudel (Berlin: Springer), 31

Flasar, F., Conrath, B., Gierasch, P., \& Pirraglia, J. 1987, JGR, 92, 15011

Fletcher, L. N., Gustafsson, M., \& Orton, G. S. 2018, ApJS, 235, 24

Friedson, J., \& Ingersoll, A. P. 1987, Icar, 69, 135

Gao, P., Marley, M. S., Zahnle, K., Robinson, T. D., \& Lewis, N. K. 2017, AJ, 153, 139

Hanel, R., Conrath, B., Flasar, F., et al. 1986, Sci, 233, 70

Irwin, P., Teanby, N., \& Davis, G. 2010, Icar, 208, 913

Irwin, P., Teanby, N., De Kok, R., et al. 2008, JQSRT, 109, 1136

Irwin, P. G., Toledo, D., Garland, R., et al. 2018, NatAs, 2, 420

Jacquinet-Husson, N., Crepeau, L., Armante, R., et al. 2011, JQSRT, 112, 2395

Karkoschka, E. 2001, Icar, 151, 84
Karkoschka, E., \& Tomasko, M. 2009, Icar, 202, 287

Kerber, F., Käufl, H.-U., Baksai, P., et al. 2014, Proc. SPIE, 9147, 91470C

Lagage, P., Pel, J., Authier, M., et al. 2004, Msngr, 117, 12

Lellouch, E., Moreno, R., Orton, G., et al. 2015, A\&A, 579, A121

Li, C., Le, T., Zhang, X., \& Yung, Y. L. 2018, JQSRT, 217, 353

Lockwood, G. 2019, Icar, 324, 77

Lunine, J. I., \& Hunten, D. M. 1989, P\&SS, 37, 151

McMillan, W., \& Strobel, D. 1992, BAAS, 24, 990

Meyer, B., Gouterman, M., Jensen, D., et al. 1972, in Sulfur Research Trends, ed. D. J. Miller \& T. K. Wiewiorowski (Washington, DC: ACS), 53

Molter, E. M., de Pater, I., Roman, M. T., \& Fletcher, L. N. 2019, AJ, 158, 13

Moses, J. I., Fletcher, L. N., Greathouse, T. K., Orton, G. S., \& Hue, V. 2018, Icar, 307, 124

Orton, G., Moses, J., Encrenaz, T., et al. 2018, in 42nd COSPAR Scientific Assembly, ed. T. Prince \& R. Lopez (Vancouver: International Conference Services, Ltd.), B5.4-3-18

Orton, G. S., Aitken, D. K., Smith, C., et al. 1987, Icar, 70, 1

Orton, G. S., Fletcher, L. N., Encrenaz, T., et al. 2015, Icar, 260, 94

Orton, G. S., Fletcher, L. N., Moses, J. I., et al. 2014a, Icar, 243, 494

Orton, G. S., Moses, J. I., Fletcher, L. N., et al. 2014b, Icar, 243, 471

Pollack, J. B., Rages, K., Baines, K. H., et al. 1986, Icar, 65, 442

Rages, K., Hammel, H., \& Friedson, A. 2004, Icar, 172, 548

Roman, M. T., Banfield, D., \& Gierasch, P. J. 2018, Icar, 310, 54

Smith, B. A., Soderblom, L. A., Banfield, D., et al. 1989, Sci, 246, 1422

Sromovsky, L. A., Karkoschka, E., Fry, P. M., de Pater, I., \& Hammel, H. B. 2019, Icar, 317, 266

Toledo, D., Irwin, P. G., Rannou, P., et al. 2019, Icar, 333, 1

Toledo, D., Irwin, P. G., Teanby, N. A., et al. 2018, GeoRL, 45, 5329

Trafton, L. M., Orton, G., Greathouse, T., Lacy, J., \& Encrenaz, T. 2012, AAS/DPS Meeting, 44, 412.21

Wallace, L. 1983, Icar, 54, 110

Yelle, R. V., McConnell, J. C., Strobel, D. F., \& Doose, L. R. 1989, Icar, 77, 439

Zahnle, K., Marley, M. S., Morley, C. V., \& Moses, J. I. 2016, ApJ, 824, 137 